\title{
Extrapulmonary tuberculosis: an old but resurgent problem
}

\author{
Ali H. Baykan ${ }^{1 *} \mathbb{0}$, Hakan S. Sayiner ${ }^{2}$, Elcin Aydin ${ }^{3}$, Mustafa Koc ${ }^{4}$, Ibrahim Inan ${ }^{5}$ and Sukru M. Erturk ${ }^{6}$
}

\begin{abstract}
Tuberculosis (TB) primarily affects the lungs, but some of its most devastating clinical consequences arise because of its ability to spread from the lungs to other organs. Extrapulmonary TB (EPTB) constitutes 15-20\% of all TB cases. Imaging findings are not always specific and can mimic many diseases; therefore, EPTB should be considered in the differential diagnosis, particularly in patients with immune system disorders (AIDS, patients receiving chemotherapy, etc.) and those in other high-risk groups including people with diabetes. The bacterium's passage to the regional lymph nodes is essential for developing a protective T-cell-mediated immune response, but the bacterium can spread hematologically and via the lymphatic system, leading to extrapulmonary involvement. Diagnosis of EPTB in high-risk patients is made based on suspected clinical and radiological findings, but further positive culture and histopathological confirmation may be required in some instances. Radiological evaluations are critical for diagnosis and crucial in planning the treatment and follow-up. This paper aims to review the typical and atypical imaging features and the differential diagnosis of EPTB.
\end{abstract}

Keywords: Tuberculosis, Extrapulmonary tuberculosis, Magnetic resonance imaging, Computed tomography, X-ray

\section{Key points}

- EPTB is not an uncommon type of TB, but it is difficult to recognize.

- EPTB usually occurs with the lympho-hematogenous spread of primary infection (during or later) to extrapulmonary organs but can sometimes develop without pulmonary involvement.

- Radiological evaluations are critical for diagnosis and follow-up.

\section{Background}

Tuberculosis (TB) is one of the infectious diseases with the highest preventable morbidity and mortality rates globally [1]. According to the World Health Organization

\footnotetext{
*Correspondence: drbaykan@yahoo.com

${ }^{1}$ Department of Radiology, Faculty of Medicine, Adiyaman University,

Yunus Emre Mahallesi 1164 Sokak No:13, 02200 Merkez, Adıyaman, Turkey

Full list of author information is available at the end of the article
}

(global TB report 2021), an estimated 10 million people fall ill with $\mathrm{TB}$, and 1.5 million die from $\mathrm{TB}$ every year [2]. High-risk groups include immunosuppressed individuals, malnourished people, people of low socioeconomic status, prisoners, alcoholics, children, the elderly, the homeless, nursing home residents, those living in TBendemic areas, and healthcare workers [3]. One in every five TB cases presents with extrapulmonary TB (EPTB) [3]. The tuberculin skin test, serum interferon-gamma release, polymerase chain reaction, adenosine deaminase assays, and imaging modalities are used to diagnose EPTB, although biopsies and culture studies remain the gold standard [3-5]. EPTB can occur almost anywhere in the body, most commonly in the lymph nodes (50\%), the pleura (18\%), the genitourinary system (13\%), bones and joints $(6 \%)$, the gastrointestinal system $(6 \%)$, the central nervous system (CNS) (3\%) and the spine (3\%) [1].

EPTB shows various clinical and radiological features depending on the organ it affects, which can often mimic other diseases [6]. Therefore, early diagnosis and treatment are essential. Radiological modalities can be used 
separately or together, depending on the affected organ and the extent of the involvement. For example, X-rays and computed tomography $(\mathrm{CT})$ are conducted if there is bone involvement, ultrasound (US), CT and magnetic resonance imaging (MRI) in cases of possible abdominal $\mathrm{TB}$, the US in the presence of a superficial lesion such as lymphadenitis, mammography, and the US if mastitis has developed, and MRI if the CNS is affected. MRI helps show whether deep tissues are affected by TB in the presence of musculoskeletal involvement. Additionally, positron emission tomography (PET)-CT can evaluate the extent of skeletal TB and monitor the response to treatment $[3,7]$. This review emphasizes radiology's role in diagnosing ЕРТВ by presenting examples of TB that involve different parts of the body.

\section{Pathophysiology}

TB is a chronic bacterial infection caused by mycobacteria called the Mycobacterium tuberculosis complex (Mycobacterium africanum, Mycobacterium microti, Mycobacterium tuberculosis, and Mycobacterium bovis). It is characterized by the presence of caseous granulomas in infected tissues. M. tuberculosis is the cause of the disease in $97-99 \%$ of cases. TB is an aerobic, acid-fast, non-motile, non-encapsulated, non-spore bacillus discovered by Robert Koch in 1882 [8]. It can involve almost all organs but is most frequently found $(80-85 \%)$ in the lungs $[1,4,9]$.

The cell-wall structure of mycobacteria is different from other bacteria; they have an intracellular lifestyle and chronic infection properties and are resistant to chemical structures including acid and alkaline environments, antibiotics, disinfectants, enzymes, and free radicals. The cell structure prevents phagolysosomal fusion formation within the macrophage, which plays a role in immunity [10].

The disease is most commonly transmitted by entering $M$. tuberculosis into the lungs via droplets from person to person or transferring $M$. bovis into the gastrointestinal tract by consuming non-pasteurized dairy products. Although rare, cases involving congenital transmission, sexual transmission, vaccination, and therapeutic installation have also been reported [11].

The TB bacillus spreads to the lung alveoli via droplets, phagocytoses by alveolar macrophages, and multiplies within macrophages. Alveolar macrophages differentiate from macrophages to histiocytes by interacting with $\mathrm{T}$ lymphocytes. Epithelioid histiocytes and lymphocytes gather in small clusters and form granulomas to restrict the disease $[12,13]$. This granuloma (with a central area of caseous necrosis) containing bacteria is called primary lesion or Ghon focus [12, 14]. If this granuloma breaks up, aerosols are formed that can infect other people via expectoration. [12].

Bacillus growth in macrophages and alveolar spaces are followed by new monocyte migration from the blood to the lesion area and the migration of macrophage-mediated bacilli to neighboring lymph nodes. The bacilli can then spread from lymph nodes to the subclavian vein and throughout the body via the hematogenous route. Using the lymphohematogenous route, the bacillus creates new infection foci until an immune response is triggered [9]. Thus, different organ involvements occur. Extrapulmonary involvement may present with or without pulmonary infection [15], and EPTB can affect any organ or tissue except for the hair, nails, and teeth. [16]. The pathogenic life cycle and extrapulmonary spread of $M$. tuberculosis are summarized in Fig. 1.

\section{Involvement of the body systems Nervous system}

CNS involvement has been found in $5-10 \%$ of patients with TB and $20 \%$ of those with AIDS-related TB [17]. It is one of the most significant causes of morbidity and mortality in TB-endemic areas. CNS-TB is usually caused by hematogenous spread, often directly from an intracranial or extracranial focus $[3,18]$. Clinical features are variable. However, a nonspecific headache, neck stiffness, fever, vomiting, radiculopathy, and even coma can appear in most cases [19]. The clinical and radiological manifestations of CNS-TB can mimic many inflammatory, infectious, and tumoral diseases.

Cranial involvement of TB may present as extra-axial (tuberculous leptomeningitis and tuberculous pachymeningitis), intra-axial (tuberculoma, focal cerebritis, tuberculous abscess, tuberculous rhombencephalitis), or tuberculous encephalopathy [3].

\section{Tuberculous leptomeningitis}

Leptomeningitis is the most common CNS-TB finding in children and adolescents and develops due to the hematogenous spread of $M$. tuberculosis. The presence of exudate, which develops in the subarachnoid space at the base of the brain, is specific to tuberculous leptomeningitis and can be seen with CT and contrast-enhanced MRI in particular (Fig. 2). The most common location is the interpeduncular fossa. In the differential diagnosis, infective meningitis and inflammatory diseases such as rheumatoid arthritis, sarcoidosis, and carcinomatous meningitis should also be considered [20].

Possible complications of tuberculous leptomeningitis include progressive hydrocephalus, vasculitis, infarction, and cranial neuropathies [3]. The most common complication is communicating hydrocephalus, which is caused by the obstruction of cerebrospinal fluid into 

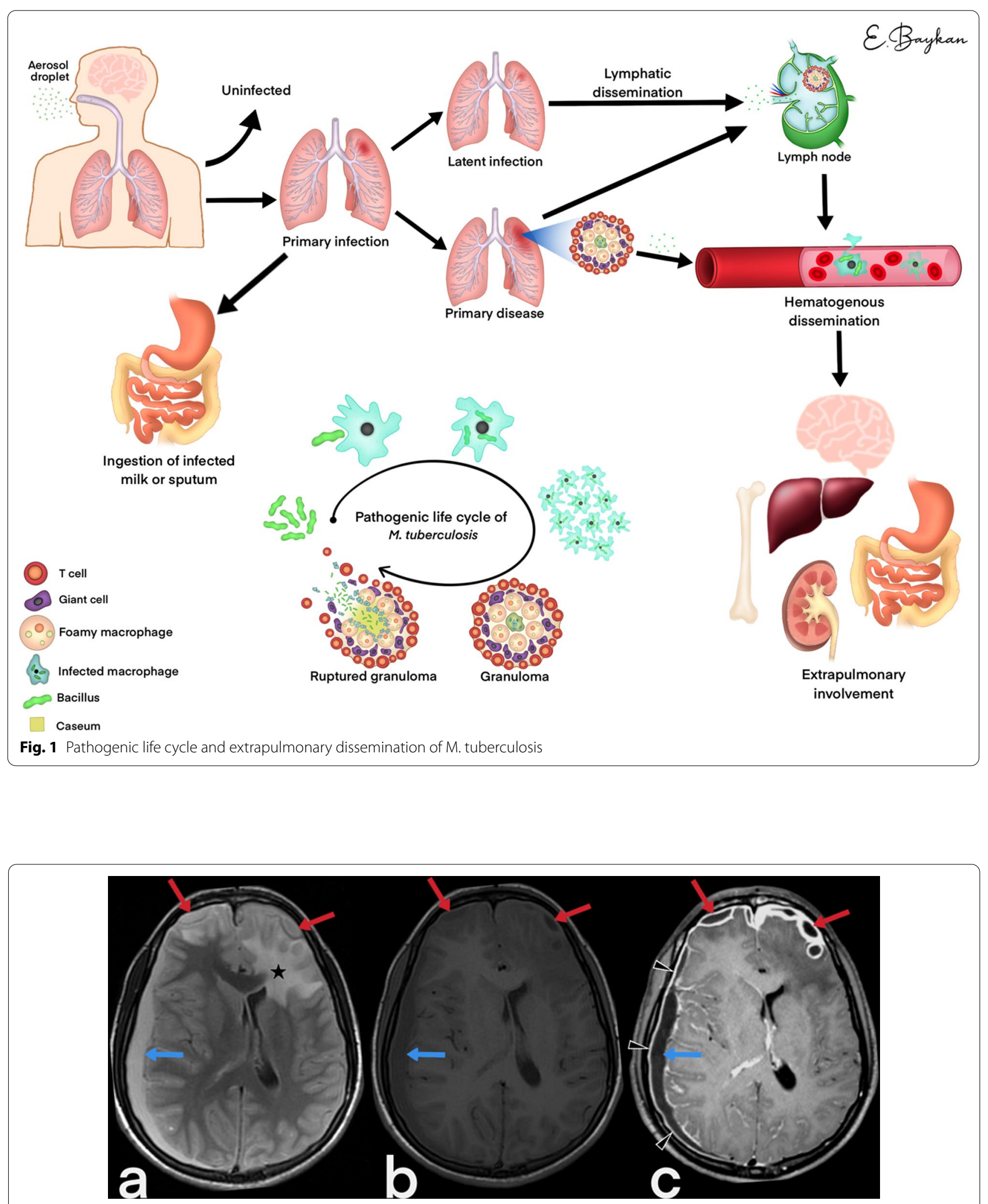

Fig. 2 Axial FLAIR (a), pre-contrast (b) axial T1 weighted, and post-contrast (c) axial T1 weighted MR images of a 21-year-old male. Axial FLAIR image shows high signal intensity caused by edema and inflammation in the left frontal lobe (star). In both anterior frontal regions (red arrows) and right parietal area (blue arrows) are seen subdural abscesses. In addition, MR images demonstrate meningeal enhancement and thickening (arrowheads) 
the basal cisternae. In some cases, non-communicating hydrocephalus caused by a tuberculoma or tuberculous abscess may also be seen [17]. Ischemic infarction may also develop as a result of cerebral arteritis. Hemorrhagic infarction caused by dural venous sinus thrombosis is another complication of tuberculous leptomeningitis. Cranial nerves 2, 3, 4, and 7 may be affected by vascular ischemia or nerve entrapment, and the diagnosis is made with nerve thickening and enhancement in MRI [21].

\section{Tuberculous abscess}

Tuberculous abscesses are rarely seen. A tuberculous abscess is either solitary or multiple lesions and is usually multiloculated with liquefaction necrosis in the central area. CT and MRI are visualized as a mass with a cystic center, surrounding edema, and a contrast-enhanced wall [21]. A tuberculous abscess is different from a tuberculoma, which presents with caseous necrosis and liquefaction in the center.

\section{Tuberculoma}

The center of a tuberculoma contains necrotic caseous material surrounded by a capsule comprising fibroblasts, epithelioid cells, Langhans giant cells, and lymphocytes. Lesions may be solitary, multiple, or miliary, and the frontal and parietal lobes are often involved. Ring-like enhancement is observed on CT, and the typical finding of a tuberculoma is a central nidus of calcification with surrounding ring-like enhancement, known as the target sign. The MRI appearance of tuberculomas varies depending on whether or not there is caseation $[17,22]$.

Non-caseating tuberculomas are solid lesions that are non-necrotic, are located in subcortical white matter, are hypointense on T1-weighted images, hyperintense on T2-weighted images, show post-contrast homogeneous enhancement, and are surrounded by vasogenic edema [17]. Caseating tuberculomas are seen in two forms as solid caseating tuberculomas (early stage) and tuberculomas with central liquefaction (late-stage). Solid caseating tuberculomas appear hypointense or isointense on T1-weighted images and hypointense on T2-weighted images caused by paramagnetic radicals that develop due to the inflammation and the capsule being stained in the form of a ring in contrast-enhanced sections (Figs. 3, 4c) [17]. In contrast, the tuberculomas with central liquefaction present centrally hyperintense signal and peripheral hypointense rim (capsule) on T2-weighted images (Fig. 4b) [20, 21]. Neurocysticercosis, fungal granulomas, pyogenic abscesses, tumors such as lymphomas, gliomas, and metastases are included in the differential diagnosis of tuberculomas [22].

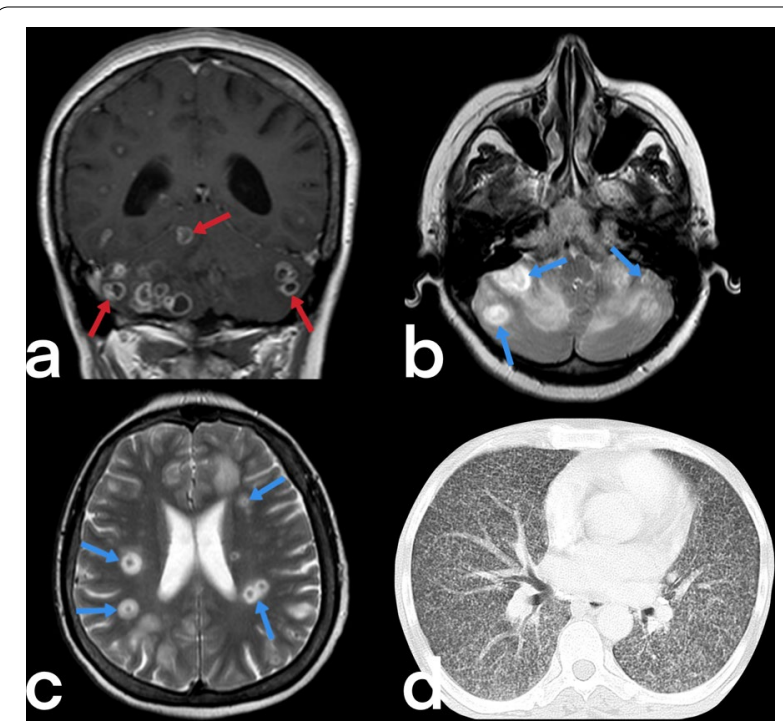

Fig. 3 Radiologic images of a 38-year-old-female who has a diagnosis of miliary TB. Multiple tuberculomas (red arrows) show peripheral rim-like enhancement on post-contrast T1 weighted image in the cerebellum (a). On T2-weighted images (b, c) demonstrate focal lesions (tuberculomas) with central isointense signal and peripheral hyperintensity in gray matter (blue arrows). Multiple nodules secondary to miliary TB are seen (d)

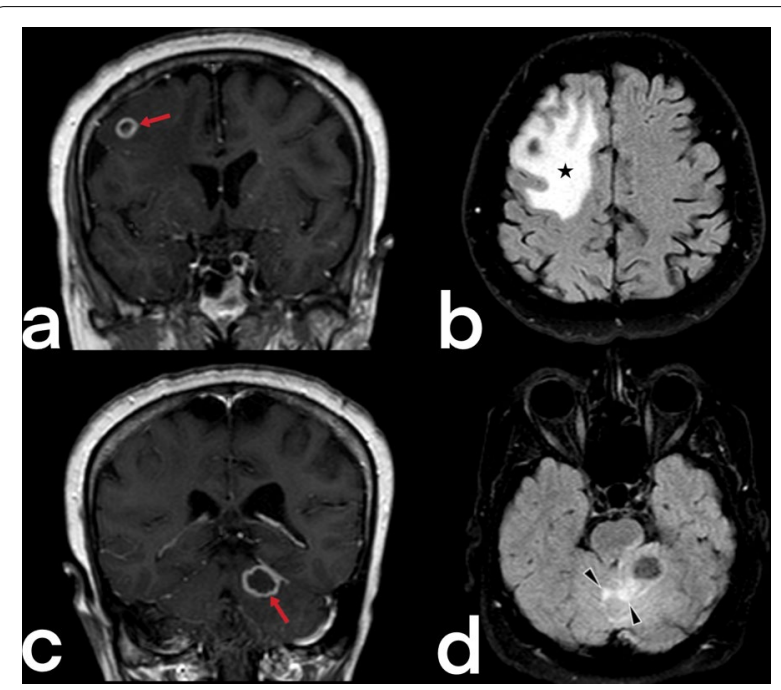

Fig. 4 MR images of a 63-year-old female with central nervous system TB. Focal lesions (arrows) with rim-like enhancement are seen (tuberculoma) on contrast-enhanced coronal T1 weighted images $(\mathbf{a}, \mathbf{c})$. Perilesional increased signals (asterisk and arrowheads) due to vasogenic edema are noted on axial FLAIR images $(\mathbf{b}, \mathbf{d})$

\section{Spinal cord TB}

Spinal cord TB can be observed in cases of intramedullary myelitis, spinal meningitis, and arachnoiditis. It develops because of the direct spread of tuberculous spondylitis from the vicinity or hematogenous spread from the 
primary focus [23]. Spinal edema and segmental infarcts may develop due to infective thrombosis and vasculitis in the spinal arteries [23]. An increase in cord signal is observed in T2-weighted MRI images caused by thick, irregular meningeal involvement (Fig. 5), spinal cord and root inflammation, myelitis (Fig. 6), edema, and infarction $[23,24]$. Syringomyelia caused by chronic arachnoiditis can also be detected in the later stages [23]. Extreme rarely (only 2 out of 1000 cases of central nervous system TB), it may present as intramedullary spinal tuberculoma (Fig. 7) [25]. Other spinal cord lesions (for example, myelitis, astrocytic glioma, ependymocytoma, and hemangioblastoma) should be considered in the differential diagnosis $[25,26]$.

\section{Head and neck}

\section{Tuberculosis of the larynx}

Laryngeal TB develops in 1 out of every 100 cases with lung TB, and almost all cases with laryngeal TB have primary lung TB. This result is explained by the direct contact of bronchogenic secretions with the laryngeal mucosa. Rarely, laryngeal tuberculosis may develop without pulmonary involvement [27]. In a patient with pulmonary TB, the lesion in the larynx should first suggest laryngeal TB. Its symptoms are hoarseness, pain, and odynophagia. Vocal folds, pre-epiglottic and paraglottic areas show soft tissue thickening and inflammatory changes (Fig. 8) [20].

\section{Tuberculous lymphadenitis}

Tuberculous cervical lymphadenitis is also known as scrofula and is the most common form of EPTB seen in endemic populations [3]. Cervical lymphadenitis may develop during primary lung infection or via lymphatic dissemination from the mediastinal lymph nodes after the latent period $[4,23]$. Although lymph nodes initially appear homogeneous (Figs. 9, 10), they may later be observed as nodular formations that show low CT density due to central necrosis. Calcification can also be seen in lymph nodes in the later stages [23, 28]. Ultrasound findings are variable. While the first finding is a roundshaped nodal enlargement in the early stage, abscess, necrosis, and fistula formation are more common in the late period (Fig. 11) [29]. Localizations of tuberculous lymphadenitis include cervical (63\%), mediastinal (27\%), and axillary $(8-10 \%)$ nodes. Most cases present with unilateral cervical lymphadenopathy [3]. The differential

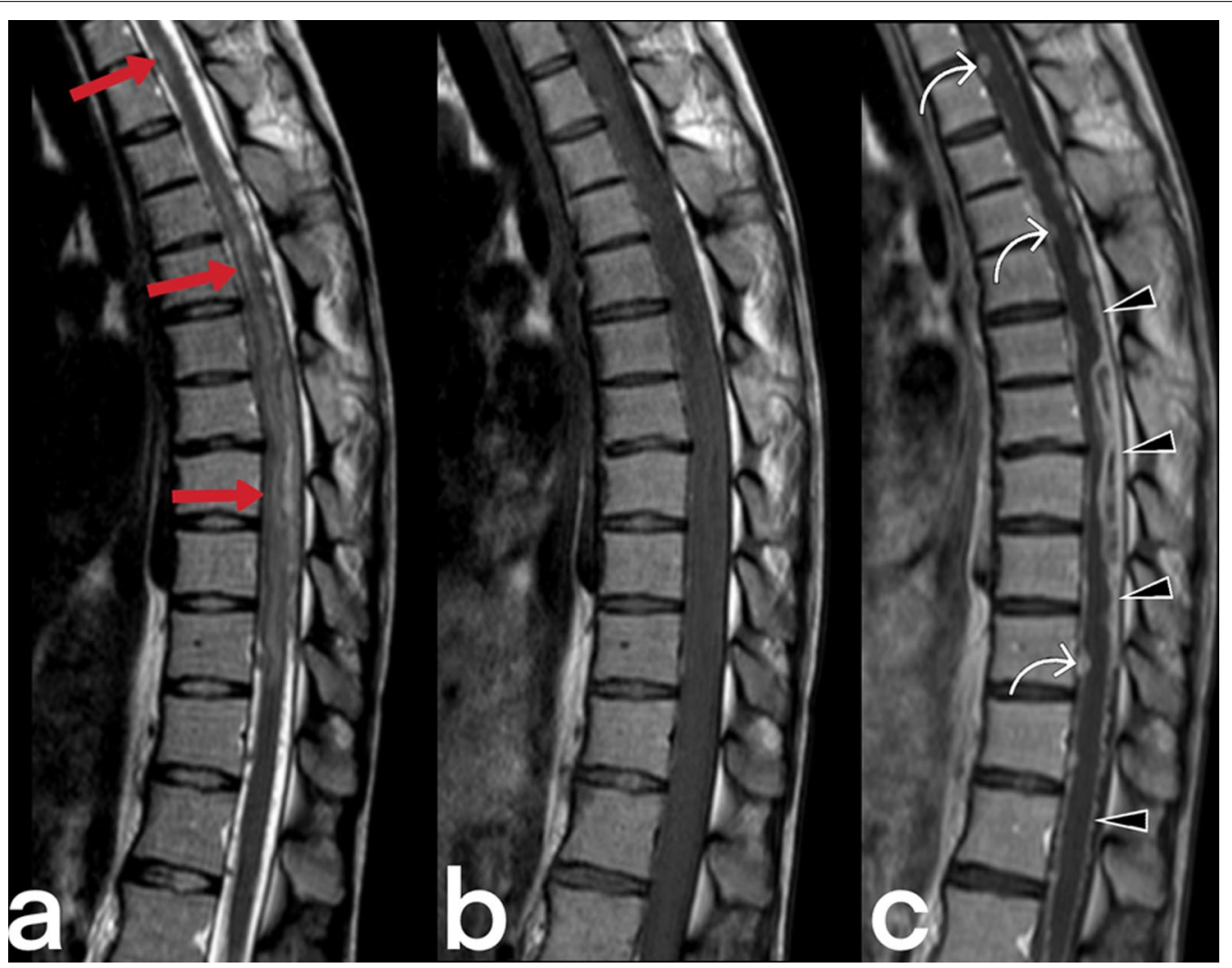

Fig. 5 MR images of thoracic vertebrae of a 23-year-old female. Sagittal T2-weighted image reveals a heterogeneous signal increase in the spinal cord (red arrows) (a). Precontrast (b) and postcontrast (c) T1 weighted images of the same patient show linear (arrowheads) and nodular (curved arrow) dural enhancement and tuberculous abscess formations in the posterior epidural space 

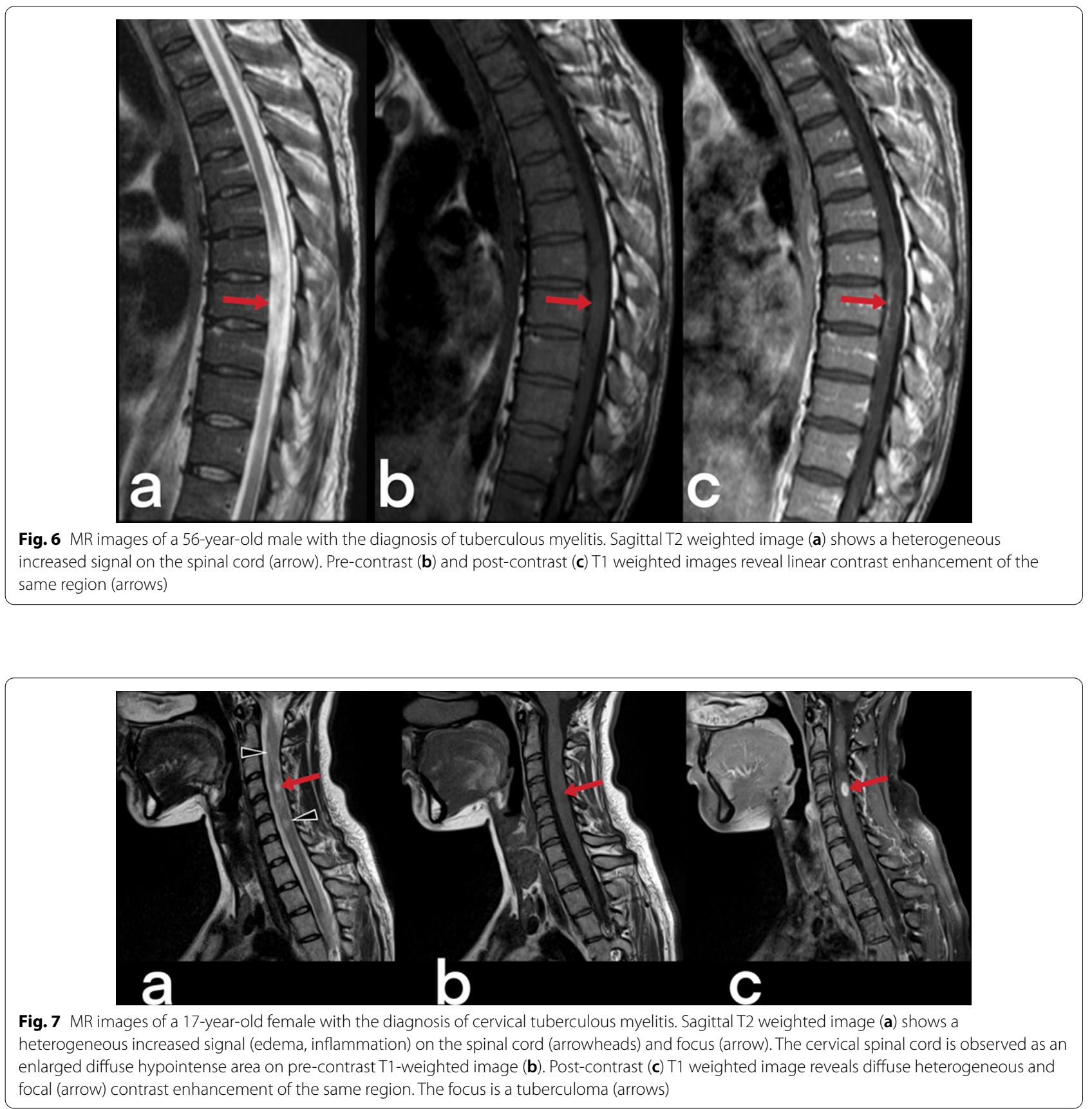

diagnosis considers the metastatic necrotic lymph nodes of papillary thyroid carcinoma and squamous cell cancer [23].

\section{Other regions}

The areas less frequently involved in the head and neck region include the temporal bone, pharynx, tonsil, sinonasal cavity, thyroid gland, skull base, lacrimal sac, bulbus oculi, and other regions of the neck [20]. Primary involvement is infrequent. It usually develops as a result of hematogenous dissemination (e.g., thyroid) after the lung infection, contact with the infected sputum, or saliva (e.g., eye globe) or through the ingestion of unpasteurized milk (e.g., tonsil, pharynx) [27, 30, 31]. It is generally observed as nonspecific inflammatory soft tissue thickening (Figs. 12, 13) and abscess (Figs. 14, 15). In advanced 


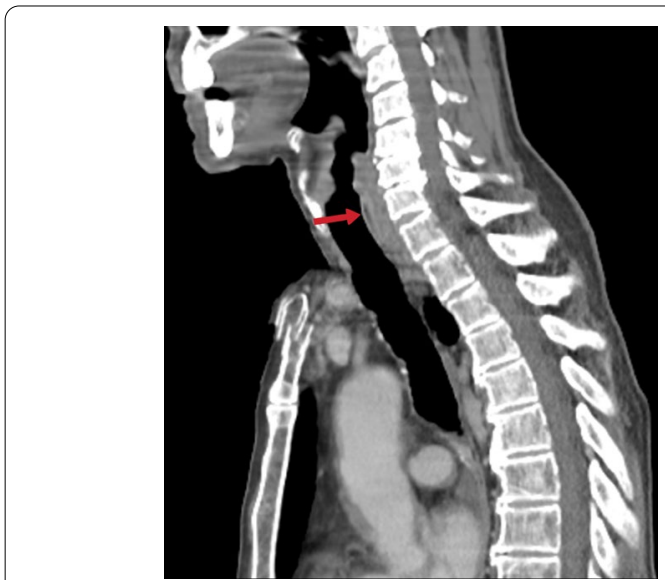

Fig. 8 A 68-year-old-male. Sagittal reformatted contrast-enhanced $\mathrm{CT}$ image reveals an increased thickness of the larynx's posterior wall (arrow). Sternum fracture is also noted in the manubrium

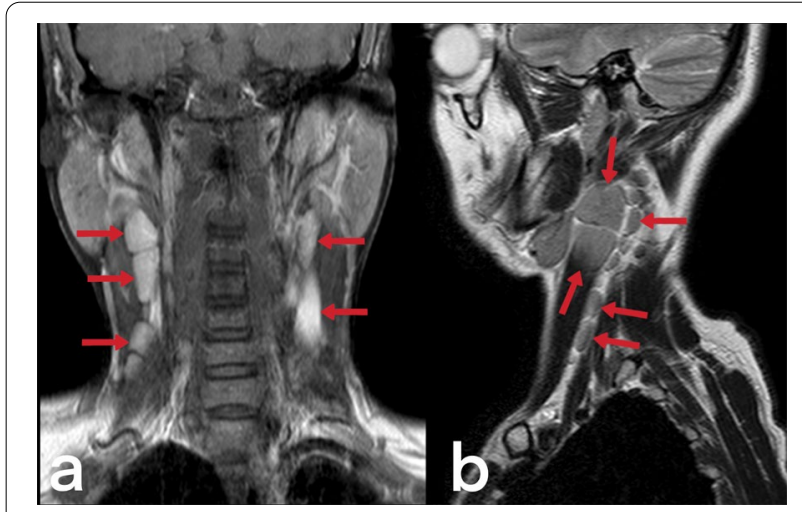

Fig. 9 Contrast-enhanced T1 weighted coronal (a) and sagittal T2 weighted (b) MR images of a 39-year-old female demonstrate multiple lymphadenopathies in the bilateral cervical chains. The patient was diagnosed with tuberculous lymphadenitis

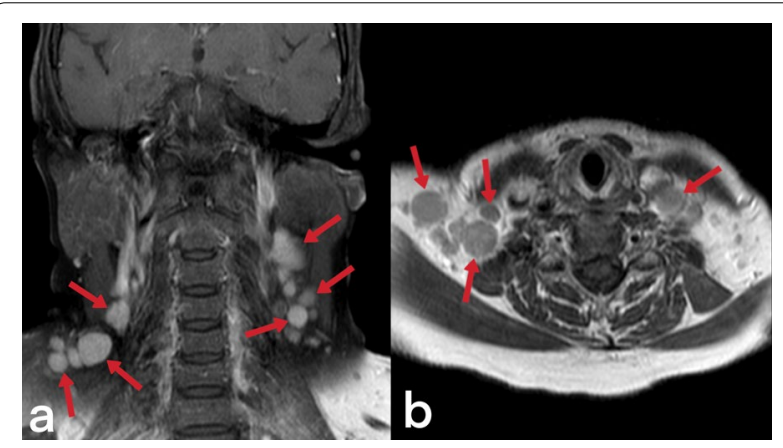

Fig. 10 Contrast-enhanced T1 weighted coronal (a) and axial T1 weighted (b) MR images of a 44-year-old female demonstrate multiple lymphadenopathies in the bilateral cervical chains and right supraclavicular region. Multiple intraabdominal lymphadenopathies are also noted (not shown)

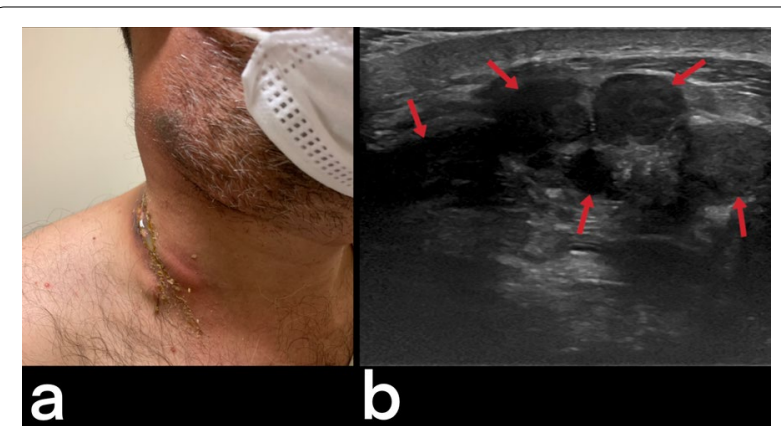

Fig. 11 A 32-year-old male. Painless, discrete, freely mobile, fistulized lumps in the neck are grown progressively (a). Ultrasound showed multiple necrotic lymph nodes (b). Scrofula of the neck

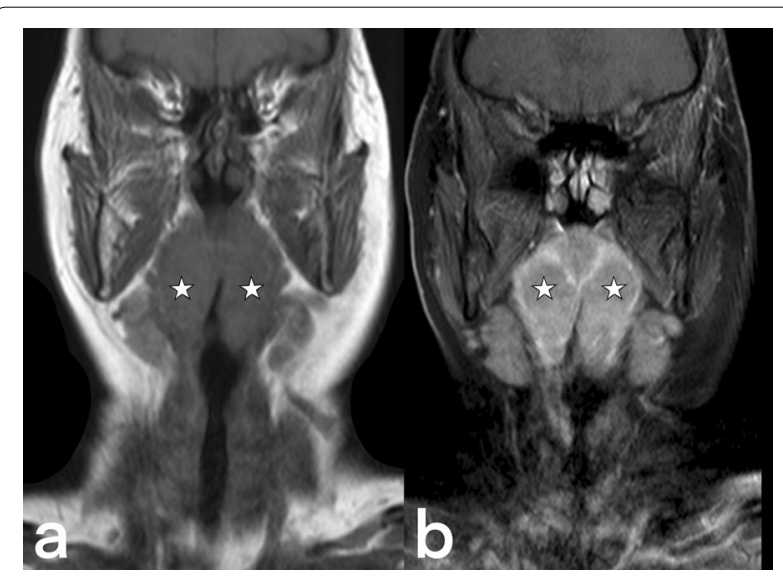

Fig. 12 A 50-year-old female. Coronal pre-contrast (a) and post-contrast (b) T1 weighted images reveal enlarged palatine tonsils, which show peripheral enhancement (stars)

cases, soft tissue masses and bone erosions can also be detected [20].

In tuberculous otomastoiditis, increased soft tissue density is observed in the tympanic cavity on $\mathrm{CT}$ in the early stages, while destruction in the middle ear structures and retroauricular/epidural abscesses can be seen in the later stages. The differential diagnosis includes pyogenic or fungal infection, sarcoidosis, cholesteatoma, and Wegener's granulomatosis [6].

Chorioretinitis and uveitis are common in ocular TB. Imaging findings show a unilateral choroidal mass, with melanoma, metastasis, hemangioma, sarcoidosis, and systemic mycosis considered in the differential diagnosis [6].

\section{Mediastinum}

Mediastinal lymphadenitis is more common in children with TB than in adults. Tuberculous lymphadenitis is 


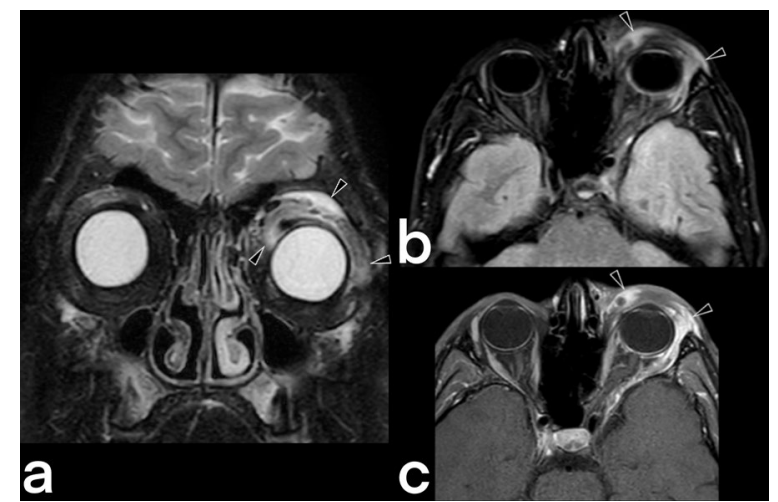

Fig. 13 A 19-year-old male patient. T2 weighted coronal (a), axial FLAIR (b), and T1 weighted post-contrast axial (c) images show periorbital involvement (arrowheads)

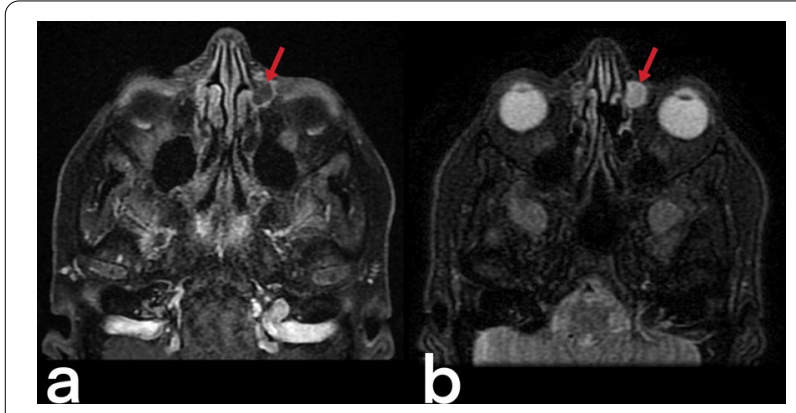

Fig. 14 A 28-year-old female patient. T1 weighted post-contrast image (a) and T2 weighted image (b) with a tuberculous abscess in the left lacrimal sac (arrows)

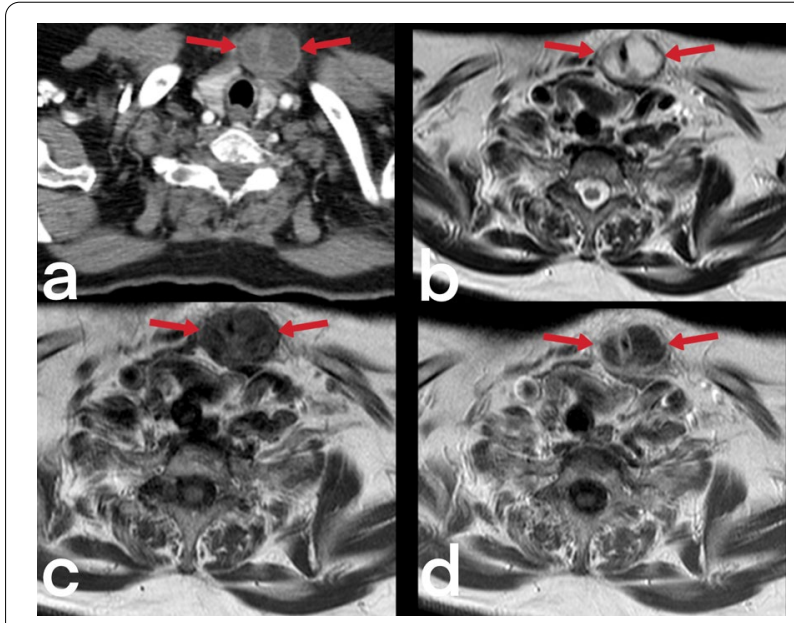

Fig. 15 Axial contrast-enhanced CT (a), axial T2 weighted (b), pre (c), and post-contrast $\mathrm{T1}$ (d) weighted images of a 69-year-old female. Biloculated abscess formation is seen in the anterior left paramedian region of the neck, which shows peripheral enhancement

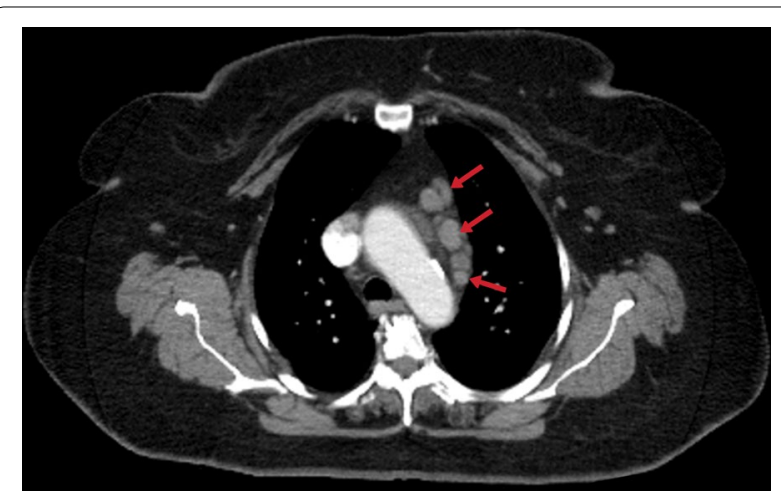

Fig. 16 Contrast-enhanced thorax CT image of a 70-year-old female. Multiple mediastinal lymphadenopathies are noted in the prevascular region (red arrows)

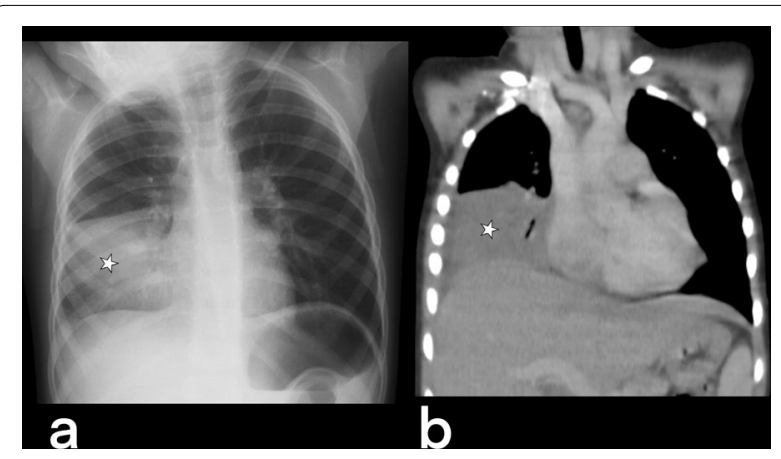

Fig. 17 The chest $X$-ray (a) and coronal reformatted CT (b) image without contrast of a 4-year-old girl demonstrate unilateral pleural effusion in the right side (stars). Tuberculous pleurisy

usually observed unilaterally in the right hilar and paratracheal area. In CT, necrotic lymph nodes larger than $2 \mathrm{~cm}$ indicate the presence of active disease (Fig. 16). Calcified lymph nodes and Ghon focus form the Ranke complex [20]. Pleural effusions or empyema can be seen in cases of TB (Fig. 17), and tuberculous empyema can sometimes mimic a thoracic wall mass (Fig. 18) [5].

\section{Cardiac}

Cardiac TB is very rare (only $0.5 \%$ of cases of extrapulmonary tuberculosis), although pericardial involvement is seen more frequently than myocardial involvement [20]. Involvement of the heart by TB can appear by hematogenous seeding from infected lung, direct spread from the adjacent focus or, via a lymphatic feed from mediastinal lymph nodes [32]. In tuberculous pericarditis, pericardial thickening $(>3 \mathrm{~mm})$, irregular contours, and mediastinal lymphadenopathy are observed. An inferior vena cava diameter of $>3 \mathrm{~cm}$, bilateral pleural effusion, intraventricular septum deformities, pericardial effusion, and localized pericardial calcification can also be observed 


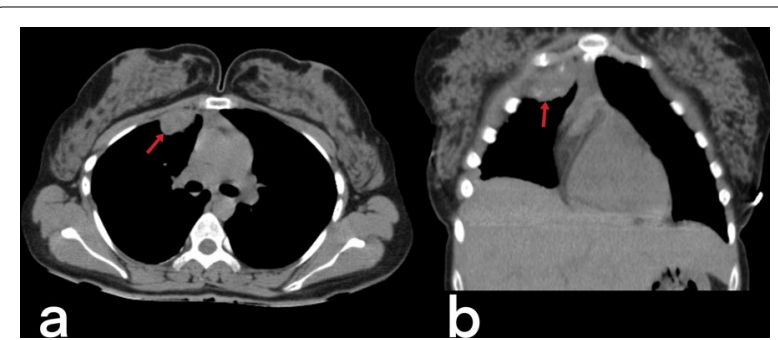

Fig. 18 Transverse (a) and coronal reformatted (b) CT images without contrast of a 24-year-old female. A mass-like focal pleural lesion in the right side containing a few coarse calcifications is noted. The patient is diagnosed with TB on histopathologic assessment after surgical removal of the mass

(Fig. 19). Myocardial involvement is asymptomatic but can be detected postmortem $[3,20]$.

\section{Breast}

Breast TB is a rare form of EPTB that affects multiparous and lactating women between 20 and 40 years old. While the spread of infection causes the primary form through abrasions in the skin of the breast or through cracks in the nipple, the secondary form can be caused by retrograde spread from infected axillary lymph nodes or by direct spread from adjacent tissue [7].

The patient's general condition is good, and systemic findings are not generally observed. The clinical presentation is nonspecific and variable, so it is difficult to distinguish clinically between granulomatous mastitis and breast cancer. However, in most cases, a painless palpable mass is often found in the breast's upper outer quadrant or central area [7].

Breast tuberculosis is radiologically observed in three forms: nodular, diffuse, and sclerosing [33]. Because females of reproductive age are affected, US is usually the first-choice imaging modality. The disease may present

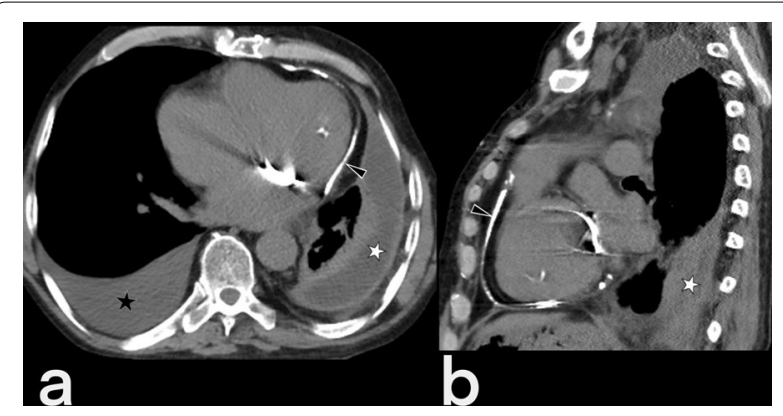

Fig. 19 Transverse (a) and sagittal reformatted (b) CT images without intravenous contrast of a 55-year-old male. Pericardial thickening with linear calcification (arrowheads), right-sided pleural effusion (black asterisk), and infected fluid collection (white asterisk) in the left pleural cavity are seen. Mediastinal and pericardial tuberculous as a circumscribed or indistinct mass. Additional findings such as abscesses, fistulas, sinus tracts, increased skin thickness/retraction can be observed, but suspicious calcifications are not generally expected (Fig. 20) [7, $34,35]$. MRI can show the extension of an abscess into extramammary areas and can also show the fistula tract's continuation into deep tissues (Fig. 21, 22) [7].

Histologically, breast TB is a form of granulomatous inflammation, so it is not easy to diagnose it pathologically. A core needle biopsy generally supports the diagnosis. Diagnosing breast TB has three essential pillars: clinical examination, radiological evaluation, and histopathological sampling [7].

\section{Abdomen}

Abdominal TB can be developed as direct involvement (via ingestion of infected lung secretions, infected unpasteurized dairy products, or infected undercooked meats) or at the time/or after the primary lung infection via spreading the lymphatic/hematologically from a focus elsewhere in the body. TB can affect all abdominal organs and tissues $[3,4]$. Clinical manifestations are nonspecific and depend on the organ involved, the extent of the affected site, and the duration of the disease (acute, chronic, or acute upon chronic). In general, clinical features may include fever (40-70\%), pain (80-95\%), weight loss (40-90\%), ascites, diarrhea, anorexia, malaise, and bowel obstruction [36, 37].

\section{Abdominal lymphadenopathy}

Abdominal lymphadenopathy is the most common abdominal TB involvement, seen in $55-66 \%$ of patients.

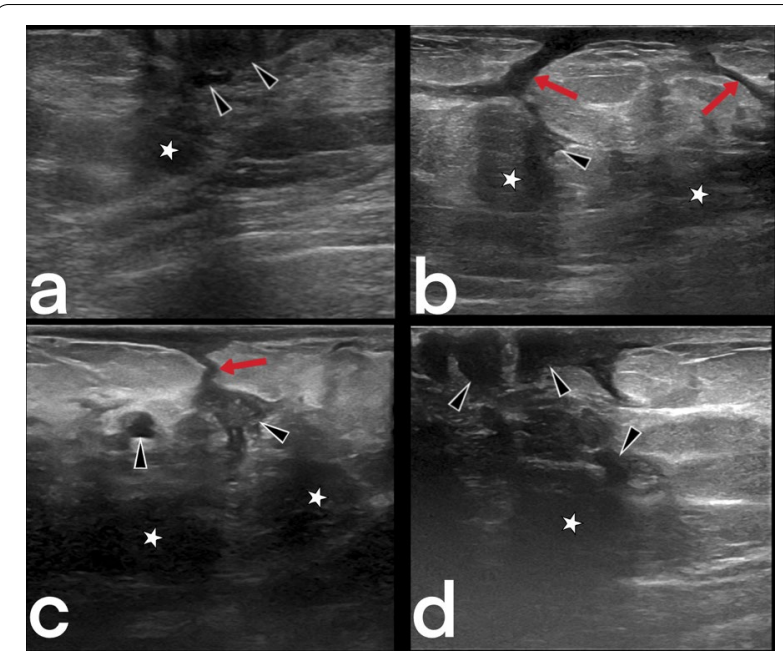

Fig. 20 Ultrasound images from four different female patients (a-d). Abscesses (arrowheads), sinus tracts (arrows), and hypoechoic parenchymal edema areas (stars) 


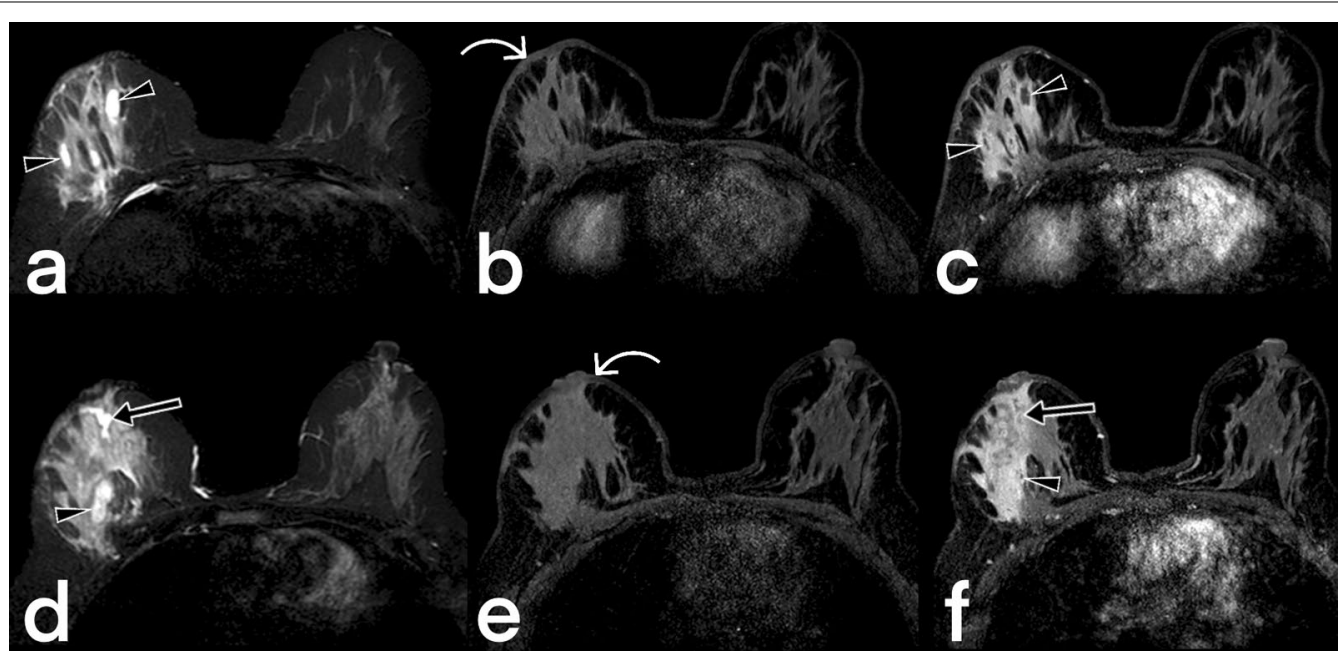

Fig. 21 MR images of a 41-year-old female. Axial T2 weighted (a, d), pre-contrast axial T1 weighted (b, e), and post-contrast axial T1 weighted (c, f) images reveal diffuse increased signal intensity, cystic lesions (arrowheads), ductal ectasia (arrow), and thickened skin (curved arrow) in the right breast. Diffuse-type breast TB

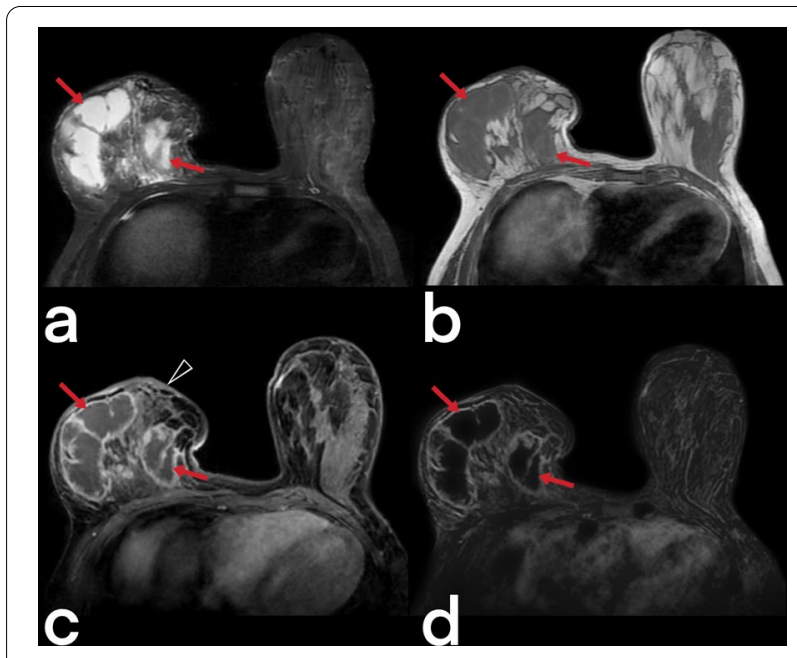

Fig. 22 Breast MR images of a 39-year-old female. T2 weighted image (a), T1 weighted image (b) post-contrast (c), and subtracted image (d) demonstrate thickening of the skin (arrowhead) and multiple abscesses (arrows) with peripheral enhancement in the right breast

CT is observed as group-forming, round-shaped, and centrally hypodense lymph nodes with and without calcification (Figs. 23, 24) [3].

\section{Peritoneal TB}

This involvement has three different forms (wet, dryplastic, and fibrotic-fixed) and is frequently associated with other abdominal TB involvements. The wet type is

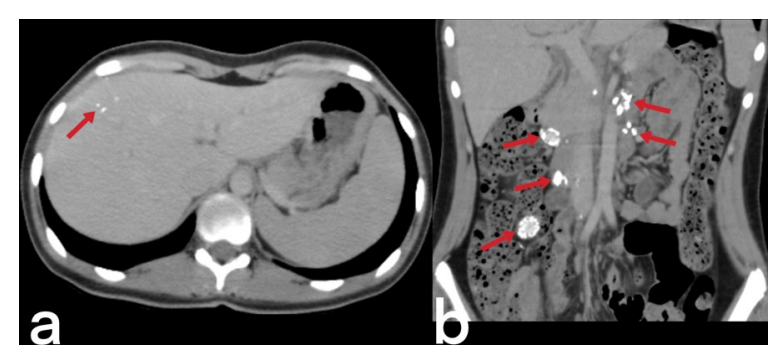

Fig. 23 Transverse abdomen CT image (a) of a 17-year-old female with known TB. Coarse calcifications in the liver parenchyma suggestive of TB sequela are noted. Coronal reformatted CT image of the same patient $(\mathbf{b})$ reveals multiple lymphadenopathies with peripheral calcifications

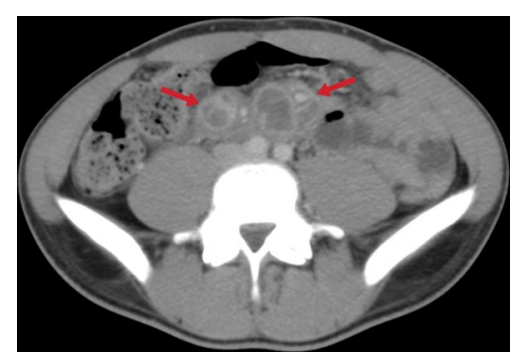

Fig. 24 A 19-year-old male. Post-contrast CT image shows multiple mesenteric lymphadenopathies with peripheral enhancement

the most common (90\%) and is observed as loculated fluid or free ascites (Fig. 25). The dry type presents the "omental cake" sign accompanied by fibrous adhesions and increased mesenteric thickness. The fibrotic type 


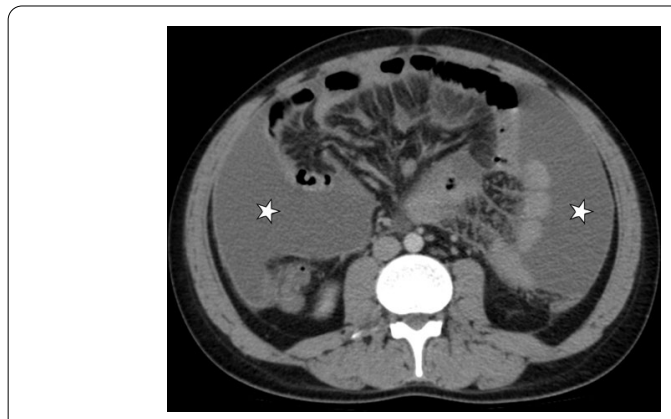

Fig. 25 Transverse CT image with intravenous contrast of a 29-year-old male. Massive ascites is indicated in the abdomen cavity (stars). The peritoneum's thin linear contrast enhancement is also noted. Tuberculous peritonitis

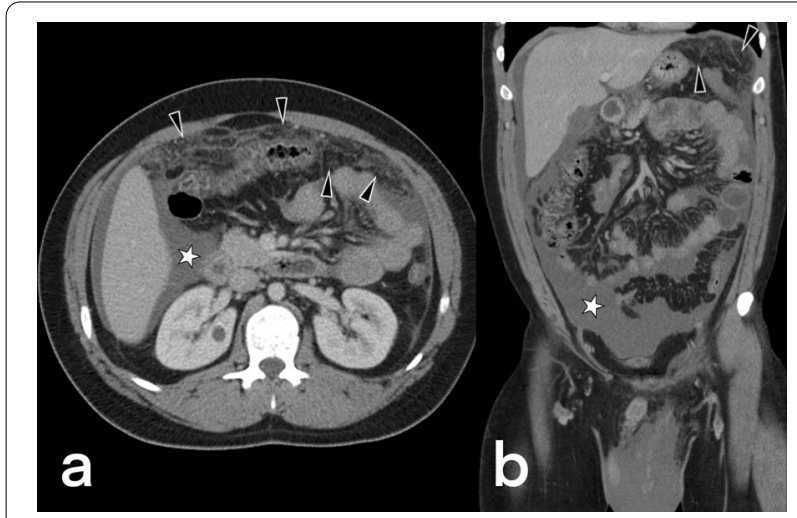

Fig. 26 A 24-year-old male patient. Axial (a) and coronal postcontrast CT (b) images show mesenteric striation (arrowheads) and ascites (star)

presents as a mass in the omentum or the mesentery, which can sometimes be confused with peritoneal carcinomatosis or malignancy [3]. Nevertheless, radiological findings are generally seen as a combination of these types (Figs. 26, 27) [38].

\section{Hepatic and splenic TB}

Although hepatosplenic TB often occurs hematogenously via the hepatic artery, it may also be caused by gastrointestinal TB spreading to the liver via the portal vein [3]. Hepatosplenic TB can be seen in micronodular (the most common), macronodular (rare), and abscess (extremely rarest) forms. The micronodular form occurs due to the spread of miliary pulmonary TB. Micronodular lesions are observed on CT as hypodense, peripherally enhanced nodules with a 0.5-2 mm diameter (Figs. 28, 29). The macronodular form is rare and consists of single or multiple lesions with a diameter of $1-3 \mathrm{~cm}$, with the imaging

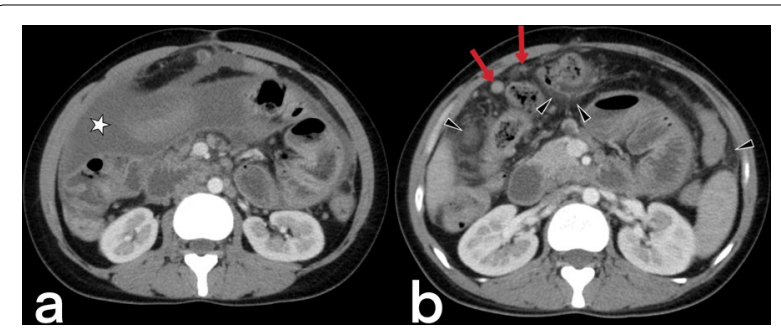

Fig. 27 A 33-year-old female. Axial post-contrast CT images (a, b) demonstrate omental nodularity, mesenteric fat stranding (arrowheads), and ascites (star). Intraabdominal lymphadenopathies are also evident (arrows) in image $\mathbf{b}$

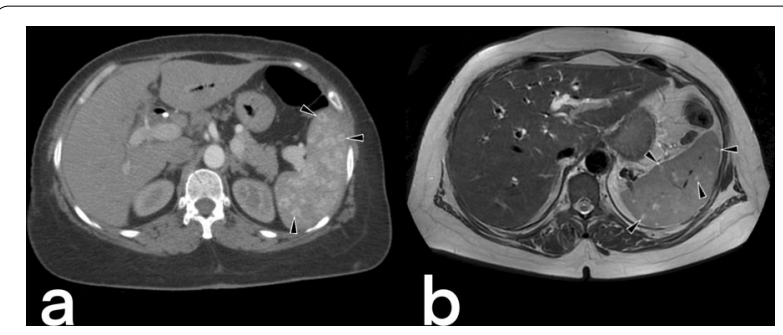

Fig. 28 Axial contrast-enhanced CT (a) and axial T2 weighted MR (b) images of a 66-year-old female demonstrate multiple foci (arrowheads) in the spleen. Note small hypodense areas with peripheral enhancement in foci (a). Splenic TB

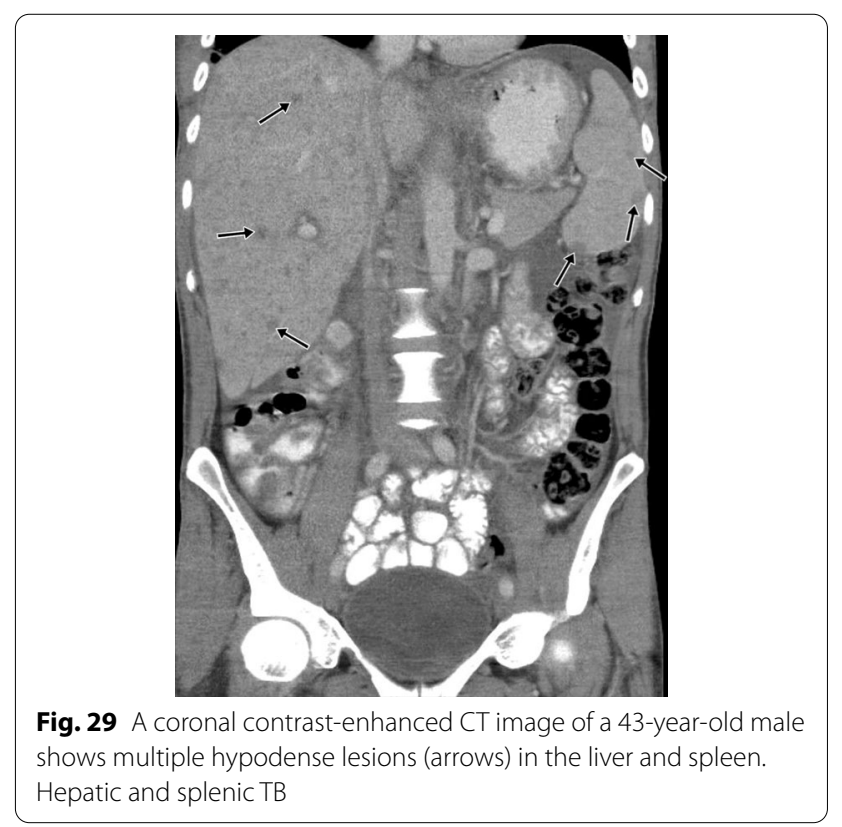

characteristics of hypointensity in the T1-weighted sequence, hypo- to hyperintensity in the T2-weighted sequence, and post-contrast peripheral and/or internal septal enhancement (honeycomb-like lesions) [3, 
39-42]. Hepatic TB abscess appears as a solitary lesion filled with bacilli, usually located in the subcapsular area, with restricted diffusion on MR and peripheral enhancement on both CT and MR (Fig. 30) [36, 39, 41, 42]. Hepatosplenic TB imaging findings are not typical for TB, and a similar appearance can be detected in metastases and abscess formations [20,36].

\section{Biliary tree $T B$}

Biliary tract involvement is an extremely rare form of EPTB. Biliary system TB originates from via portal vein (hematogenously) or periportal an infected lymph node $[36,39,43]$. Most cases of gallbladder TB also have gallstones [43]. Generally, multiple focal strictures and post-stenotic dilatations (Fig. 31) appear in the biliary tree (on US, MRI, and MRCP) [39].

\section{Pancreatic TB}

Pancreatic TB is very rare. Caseous necrosis in the pancreatic head and body is a focal, hypodense peripherally enhanced lesion with enlarged adjacent lymph nodes (Fig. 32). Neoplastic and infectious diseases are also considered in the differential diagnosis, meaning that a diagnosis is usually made based on the biopsy result $[36,42]$.

\section{Gastrointestinal TB}

The terminal ileum is the most frequently affected area in the gastrointestinal tract (up to $90 \%$ of cases of gastrointestinal TB) due to a relative abundance of adjacent

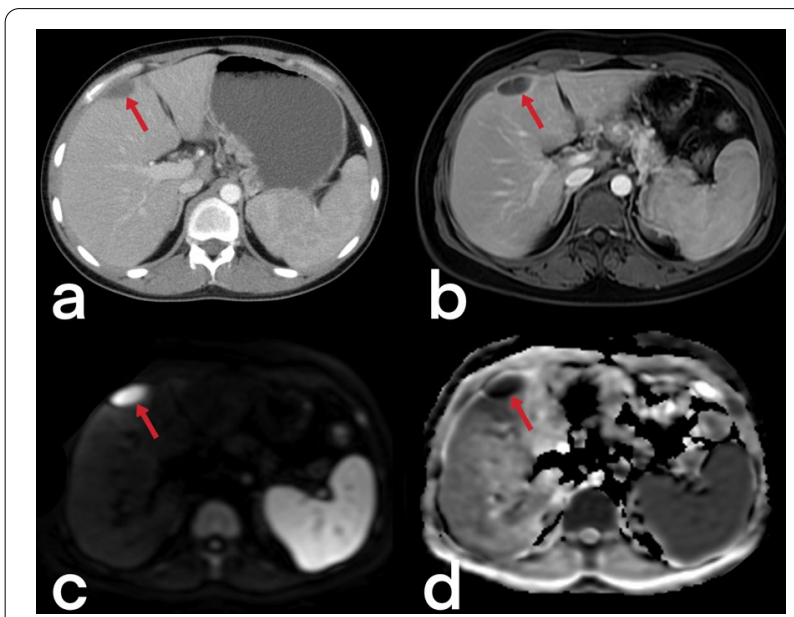

Fig. 30 Abdominal CT scan and MR images of the same patient (in Fig. 27). Contrast-enhanced CT (a) and T1 weighted contrast-enhanced MR images (b) show a small abscess formation with rim-like enhancement in the anterior subcapsular region of the liver. Diffusion-weighted images (DWI) (c) and apparent diffusion coefficient (ADC) map (d) reveal diffusion restriction consistent with an abscess

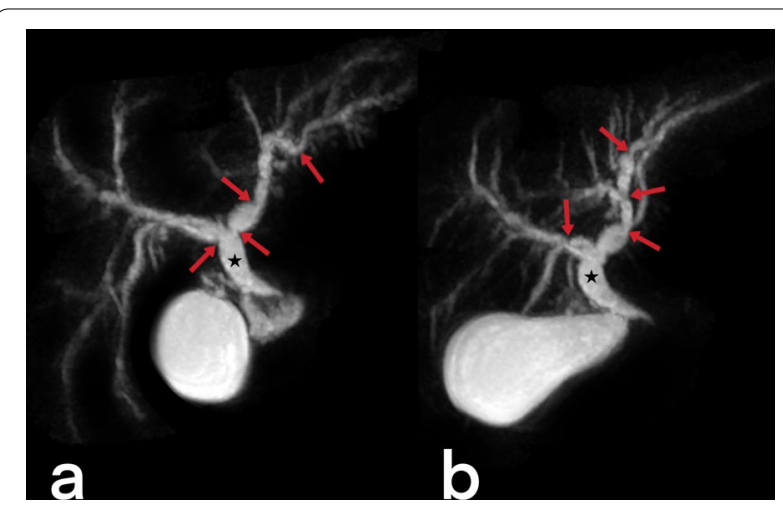

Fig. 31 A 35-year-old male. Magnetic resonance cholangiopancreatography (MRCP) images $(\mathbf{a}, \mathbf{b})$ show extensive multifocal intrahepatic biliary strictures (arrows) and dilated common hepatic duct (star)

lymphoid tissue [42]. Intestinal involvement occurs in three ways: ulcerative (most common), hypertrophic, or ulcero-hypertrophic [42, 44]. CT can detect asymmetric wall thickening and mesenteric lymphadenopathy in the terminal ileum and cecum, with a thickening of the valve lips and narrowing of the terminal ileum (the Fleischner sign) also frequently observed (Figs. 33, $34)$. In the advanced stages of the disease, symmetrical annular strictures (napkin ring), obstruction, fibrosis, irregular contours, fistulas, sinus tracts, retraction, and pouch formation can occur [23, 40]. Complications such as obstruction, perforation, and fistula formation can develop in gastrointestinal TB, while anal fissures, fistulas, or perirectal abscesses can be seen with rectal involvement [23, 40, 42]. The differential diagnosis should also include Crohn's disease, carcinoma, lymphomatous

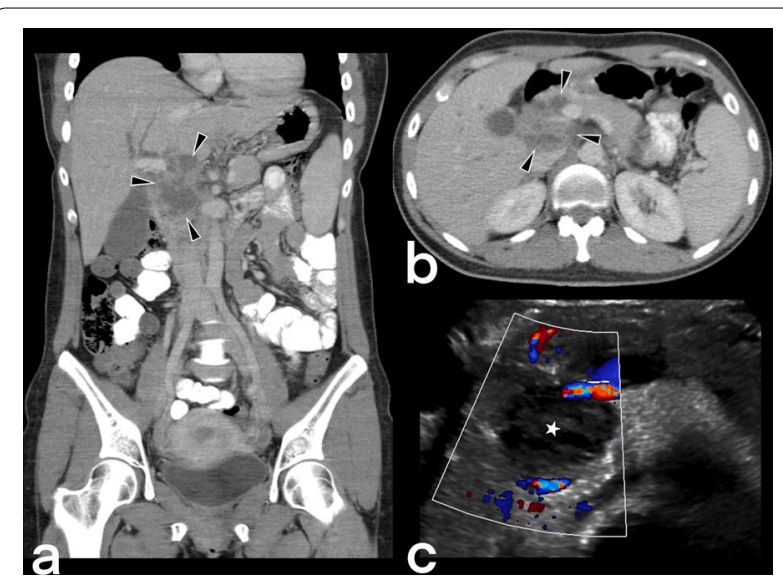

Fig. 32 A 40-year-old female. Contrast-enhancement CT scan $(\mathbf{a}, \mathbf{b})$ and Doppler ultrasonography $(\mathbf{c})$ demonstrated a large heterogeneous cystic-necrotic mass in the head of the pancreas 


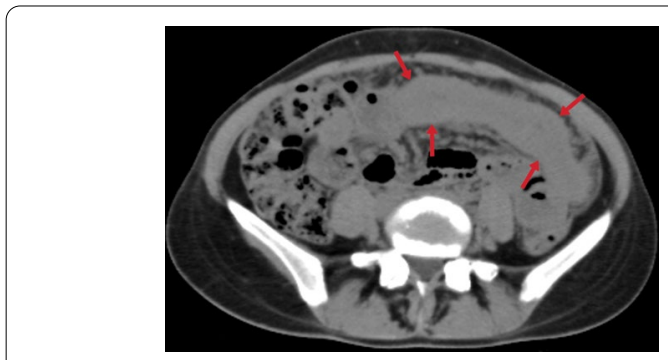

Fig. 33 Transverse CT image without intravenous contrast of a 24-year-old female. Diffuse-symmetric wall thickening of the ileal segment is noted (arrows). Ileal TB

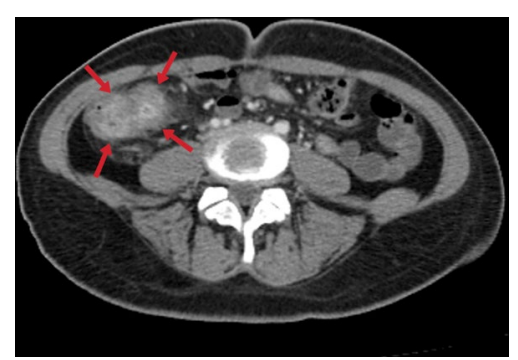

Fig. 34 A 31-year-old female. Contrast-enhanced CT image demonstrates diffuse-symmetric wall thickening and enhancement of the cecum with surrounding inflammatory changes (arrows)

involvement, amebiasis, Yersinia infection, and actinomycosis [44].

\section{Adrenal TB}

Adrenal gland involvement constitutes $6 \%$ of EPTB cases, with bilateral involvement often observed. Because of caseous necrosis, a patient can present clinical findings similar to Addison's disease (i.e., enlarged gland parenchyma with central hypodensity and peripheral enhancement) (Fig. 35) [3, 20, 23, 40].

\section{Genitourinary system}

Genitourinary TB is a type of EPTB caused by the hematogenous spread of the disease. It is more common in immunosuppressed patients and older males [36]. The patient's general condition is good and constitutional symptoms such as fever, weight loss, fatigue, and anorexia are not generally observed. The disease may be asymptomatic for a long time. Symptoms may include flank or pelvic pain, dysuria, hematuria, sterile pyuria. If female patients' genital organs are affected, menstrual irregularities, chronic lower abdominal pain, abnormal vaginal discharge, and postmenopausal bleeding may occur $[4,45$, 46]. M. tuberculosis bacillus causes abscesses in periglomerular capillaries, but these abscesses are restricted and transformed into inactive granulomas in healthy immune systems. When immunity is suppressed, the tuberculous granulomas are reactivated and spread to adjacent papillae. They pass from the papillae to the collecting system and spread to the distal genitourinary system, causing multifocal active inflammation followed by fibrosis, scarring, and calcification $[47,48]$. In genitourinary TB, lobar calcification can be detected in plain film radiography, while intravenous urography may reveal decreased calyx acuity, papillary necrosis, irregular caliectasis without pelvic dilatation, and urothelial thickening. Complications of genitourinary TB include tuberculous interstitial nephritis, sinus tracts, fistulae, and amyloidosis [49].

\section{Renal TB}

Renal TB is generally unilateral. Intravenous urography shows a moth-eaten appearance caused by erosions in the calyx and can also show papillary necrosis, hydronephrosis with irregular contours, and contrast filling debris defects $[20,23,40,48]$. CT can detect focal caliectasis due to infundibular strictures, renal calcification, parenchymal scarring, and hypodense parenchymal lesions (Fig. 36). In end-stage TB

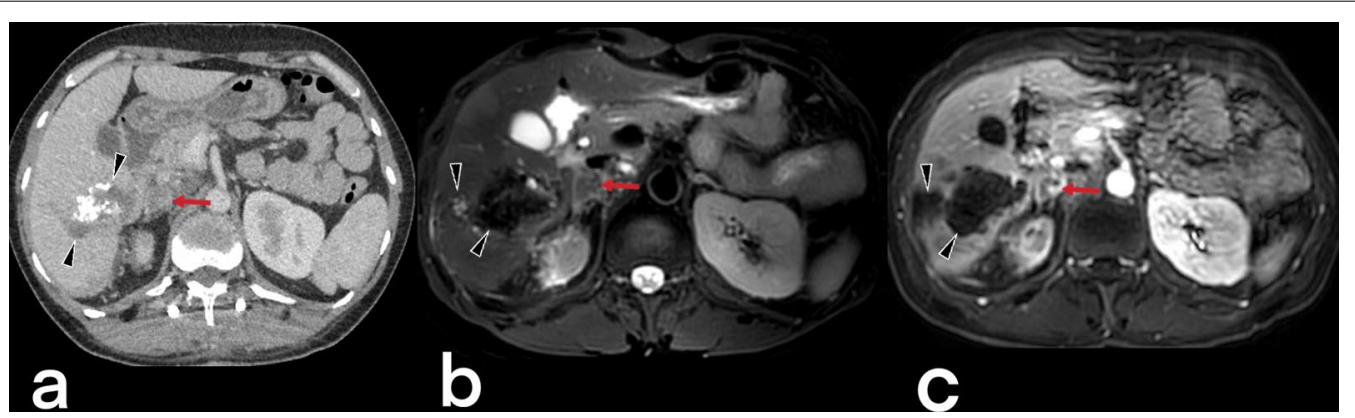

Fig. 35 A 52-year-old female. Contrast-enhancement CT (a), T2 weighted MR (b), and contrast-enhancement T1 weighted MR (c) images demonstrate a heterogeneous lesion with peripheral enhancement (arrows) in the right adrenal gland (adrenal TB). Multiple calcified heterogeneous areas (arrowheads) are also seen in the liver (hepatic TB) 


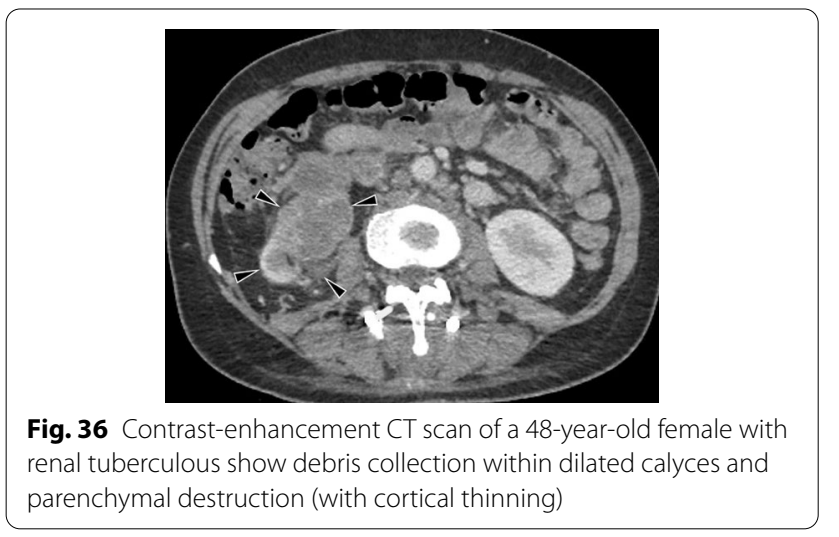

(auto-nephrectomy), calcifications with lobar distribution are pathognomonic [20].

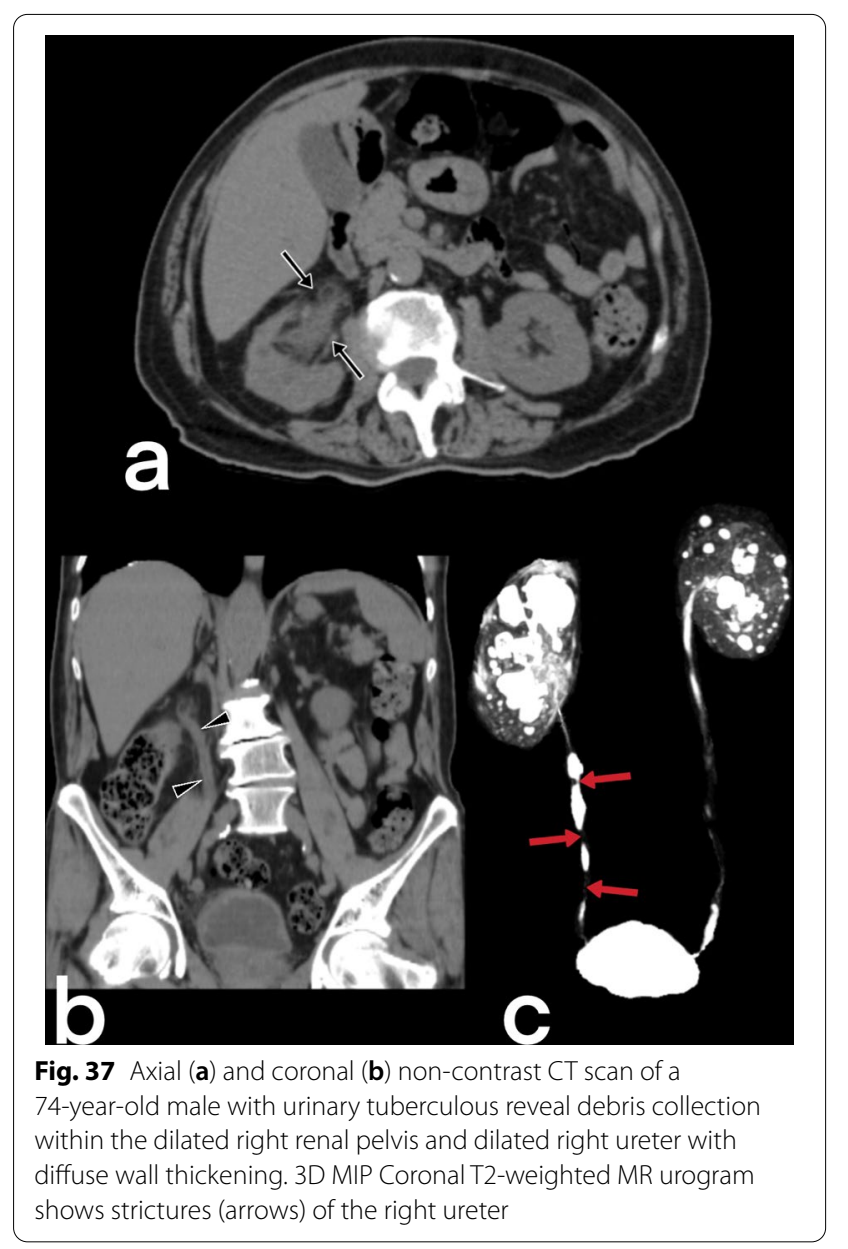

\section{Ureteral TB}

Thickening of the ureter wall is observed in acute TB involvement, while strictures in the lumen and a shortening of the ureter are detected in chronic TB involvement (Fig. 37) [20, 23, 40, 48].

\section{Urinary bladder TB}

Urinary bladder involvement is caused by the spread of the infection from the kidneys. Bladder wall thickening, ulceration, and filling defects due to granulomatous material are observed in acute TB (Fig. 38), while decreased bladder capacity and irregular and calcified appearances in bladder contours indicate chronic TB [20, $23,40]$. Fibrotic changes can also cause hydroureteronephrosis [20].

\section{Involvement of the genital organs}

Genital TB affects both men and women and can cause infertility $[23,50]$. Involvement of the prostate, seminal vesicles, epididymis, and testicles can be seen in men, while women can present with ovary, fallopian tube, and endometrium involvement. Primary genital TB can develop in women's cervix, vagina, or vulva. The transmission route can be through infected semen from male partners with active genitourinary $\mathrm{TB}$ or through the sputum used as a sexual lubricant of their partners with pulmonary TB [23]. Imaging findings can include prostatitis (linear, low-signal areas in the peripheral zone of the prostate in T2-weighted MRI images; prostate cancer is also considered in the differential diagnosis) (Fig. 39), prostate abscesses (restricted diffusion finding in MRI), epididymo-orchitis (dimensional increase and heterogeneity in the epididymis and testicles) (Fig. 40), and

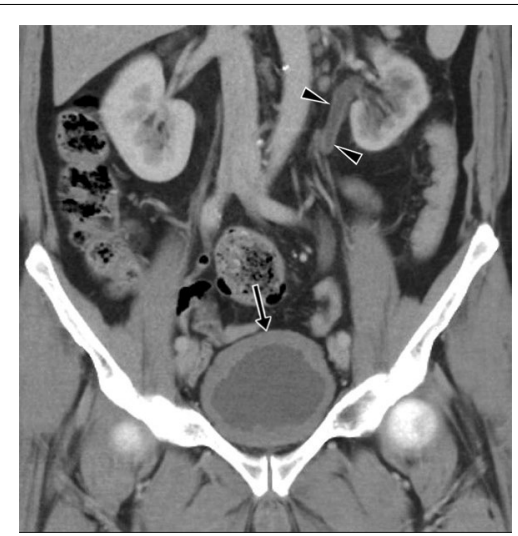

Fig. 38 A 47-year-old male. Coronal post-contrast CT scan shows diffuse thickening of the bladder wall (arrow) and dilated left proximal ureter (arrowheads). Urinary TB 


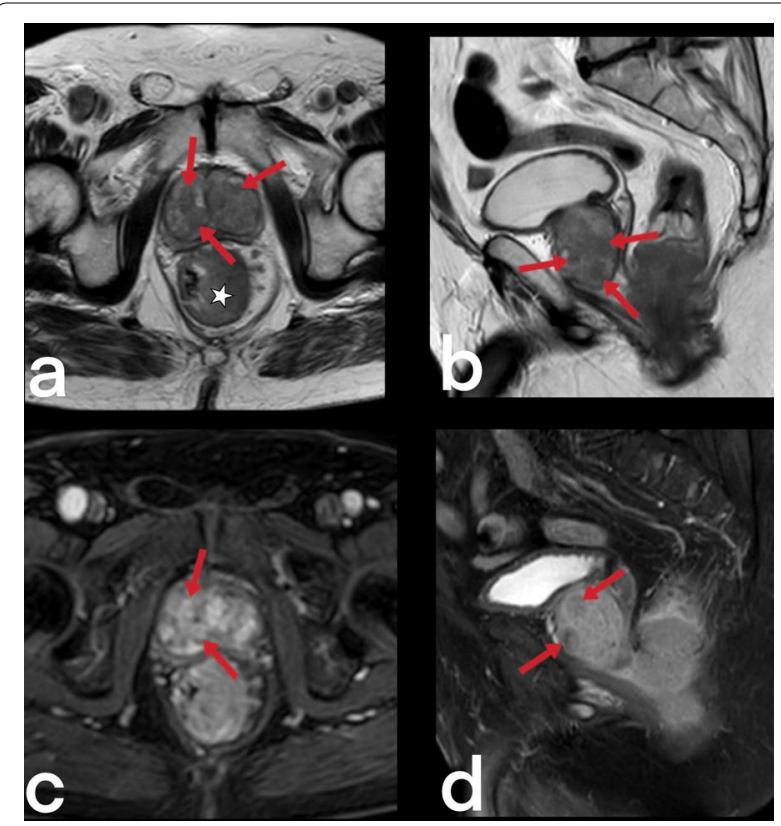

Fig. 39 Tuberculous prostatitis (abscesses) in a 53-year-old male. T2 weighted axial (a) and sagittal (b) MR images demonstrate focal, hypointense lesions within the prostate gland (arrows). Contrast-enhancement T1 weighted axial (c) and sagittal (b) MR images show heterogeneous lesions with peripheral enhancement (arrows). A rectal tumor (star) is also seen

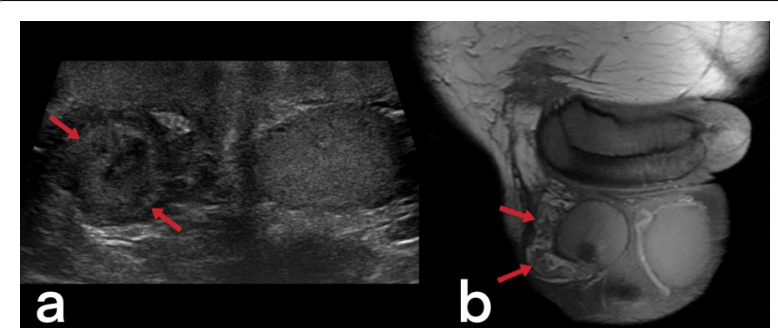

Fig. 40 A 38-year-old male. Scrotal US image (a) and post-contrast coronal T1 weighted MR image (b) reveal diffuse heterogeneity, deformation, and dilated cystic lump with peripheral enhancement in the right epididymis (arrows)

calcifications in the prostate and seminal vesicles [23, 40]. In women, salpingitis, hydrosalpinx, tubo-ovarian abscesses (Figs. 41, 42), synechiae in the fallopian tubes, ascites in the pelvic peritoneal cavity (Fig. 43), and adhesions in the endometrium resulting from TB may cause infertility [23, 48]. Differential diagnosis includes ectopic pregnancy, pelvic inflammatory disease, endometriosis, ovarian malignancy, endometrial carcinoma, and dysfunctional uterine bleeding [46].

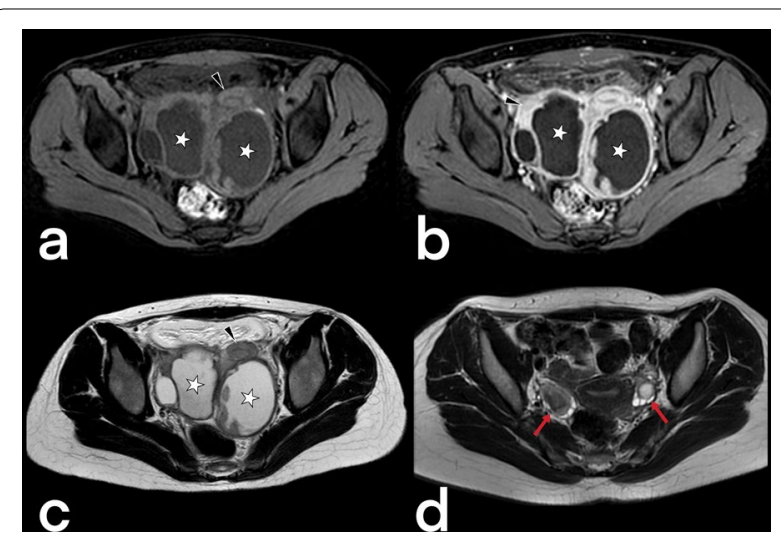

Fig. 41 Bilateral tubo-ovarian TB in a 15-year-old-female with pelvic pain. Axial pre-contrast T1 weighted (a), post-contrast T1 weighted (b), and T2 weighted (c) MR images show bilaterally enlarged ovary (stars) and dilated fallopian tubes (arrowheads). On T2-weighted MR image obtained after antituberculous therapy of 10 months, bilateral ovaries (arrows) and fallopian tubes (not shown) appear normal (d)

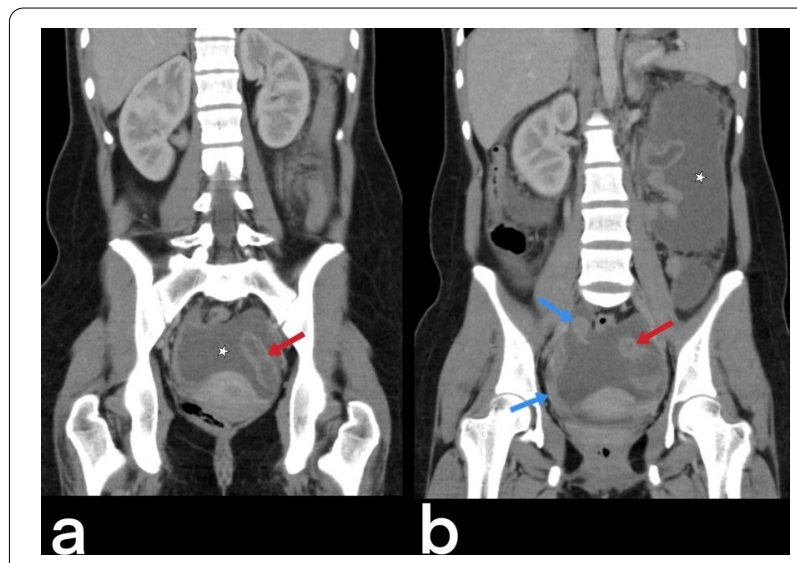

Fig. 42 Bilaterally hydrosalpinx (right: blue arrows, left: red arrow) and ascites (stars) in the peritoneal cavity are observed on coronal contrast-enhancement $\mathrm{CT}$ images $(\mathbf{a}, \mathbf{b})$ of the same patient (in Fig. 41)

\section{Musculoskeletal system}

Musculoskeletal TB is seen in 1-3\% of all TB cases and is usually caused by the hematogenous spread of $\mathrm{TB}$ or after trauma. Musculoskeletal TB is grouped as tuberculous spondylitis (Potts' spine), peripheral tuberculous arthritis, osteomyelitis, and soft tissue TB, including tenosynovitis, abscesses, and bursitis [3].

\section{Tuberculous spondylitis}

The spine is the most common bony TB involvement site, with the lower thoracic and upper lumbar vertebrae being most frequently affected. Vertebral 


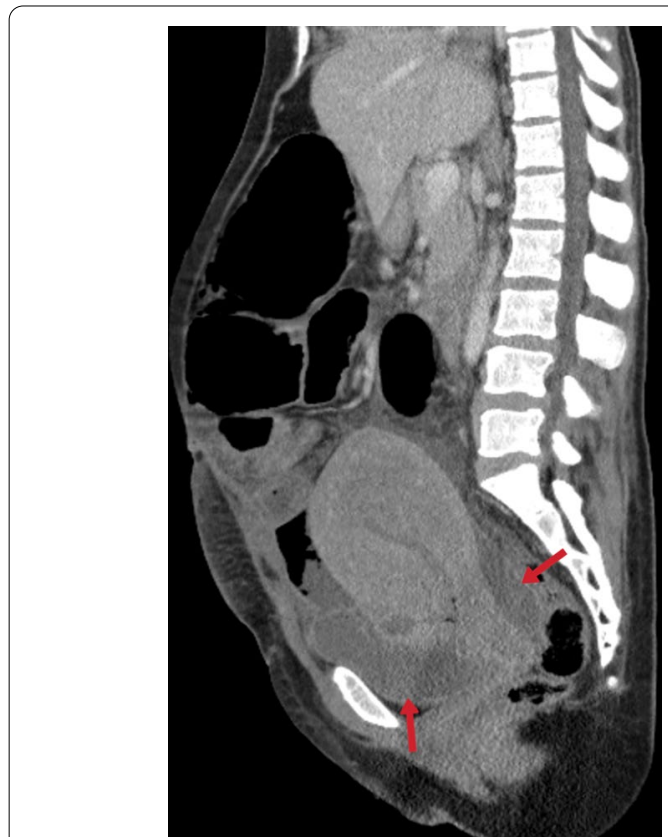

Fig. 43 Sagittal-reformatted abdomen CT image of a 22-year-old female with lower abdominal pain and fever obtained after $\mathrm{C} / \mathrm{S}$ surgery. The uterus is enlarged due to prior pregnancy. Free fluid is noted in the pelvis (arrows). Tuberculous pelvic inflammatory disease

body involvement adjacent to the disc, subligamentous involvement, and spread to the superior or inferior vertebrae without any effect on the vertebral disc are observed [3, 40]. MRI is helpful in early-stage

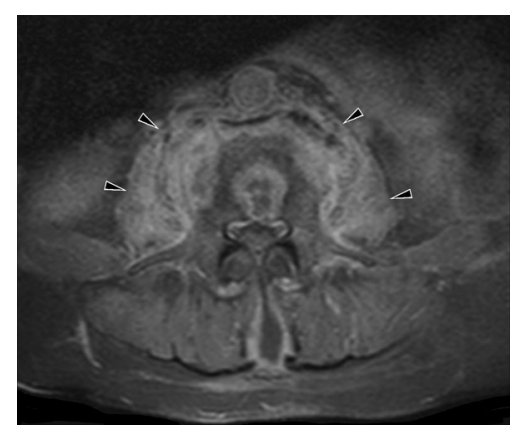

Fig. 44 Contrast-enhanced T1 weighted axial MR image of a 52-year-old male appears an inflammatory mass lesion within the adjacent soft tissues to the body of the fourth lumbar vertebra be caused by a phlegmon and abscess

diagnosis, with typical radiological findings showing hypointensity on T1-weighted images, hyperintensity on T2-weighted images, and post-contrast irregular, patchy enhancement in the anterior part of the vertebra corpus end platelets indicating bone marrow edema (Fig. 44). Subligamentous spread, a paraspinal localization, a smoothly circumscribed abscess containing a small amount of edema around three or more vertebrae (cold abscess), less bone destruction despite significant paraspinal involvement, and relative preservation of the intervertebral disc are all significant indications of tuberculous spondylodiscitis (Figs. 45, 46) [3, 23]. In untreated cases, vertebral collapse and anterior angulation can cause kyphosis, the Gibbus deformity [20].

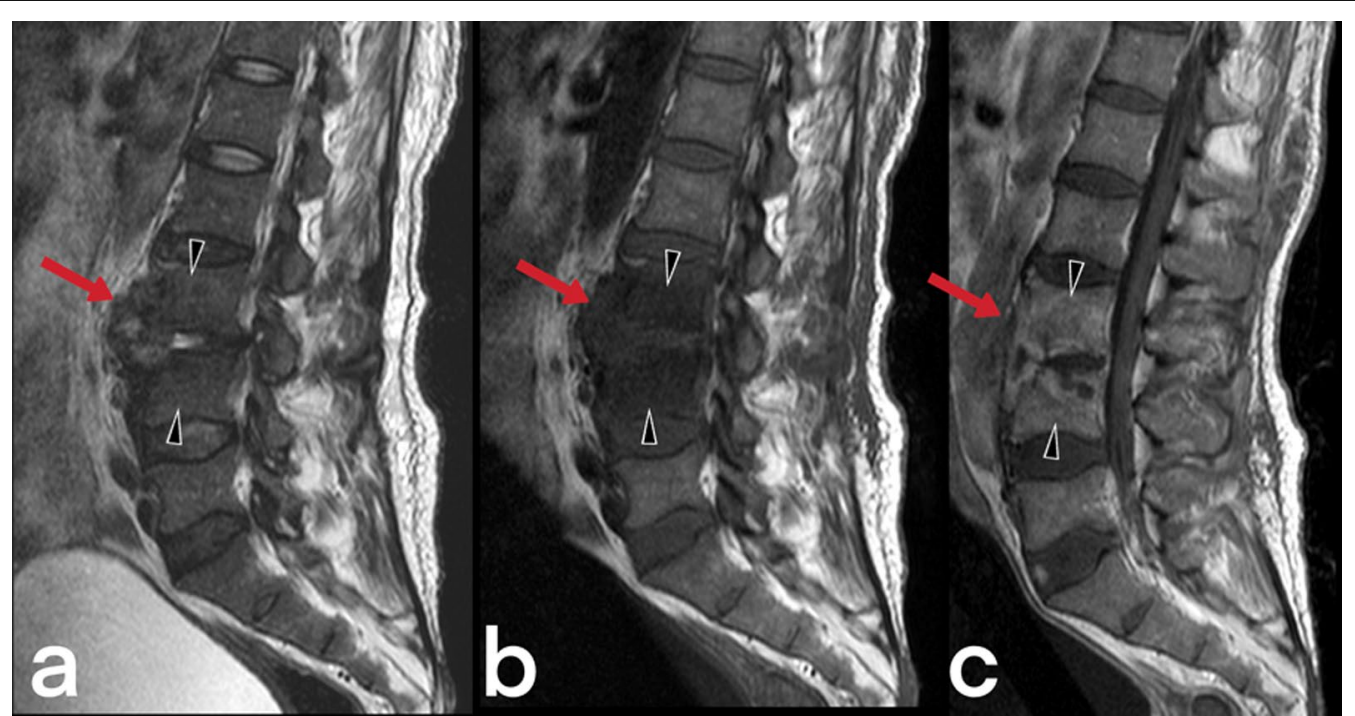

Fig. 45 MR images of lumbar vertebrae of a 47-year-old male with tuberculous spondylodiscitis. Sagittal T2-weighted image (a) shows the heterogeneous low signal in the anterior parts of the $L 3$ and $L 4$ vertebral bodies and $L 3-4$ intervertebral disc, which offers minimal anterior subligamentous extension (arrows). Pre-contrast (a) and post-contrast (b) $\mathrm{T} 1$ weighted images reveal diffuse contrast enhancement of both vertebral bodies in keeping with spondylodiscitis (arrowheads) 
The extravertebral involvement of TB is rare (1-2\%), with peripheral arthritis, osteomyelitis, tenosynovitis, and bursitis observed in the order of decreasing frequency [23]. The disease spreads through the joint space through hematogenous subsynovial vessels or the epiphysis in adults and the metaphysis in children. This involvement manifests as trabecular and cortical destruction, periosteal reaction, and a soft tissue mass $[23,40]$. Other pyogenic infections (such as brucellosis, fungal infection), metastatic tumors, and sarcoidosis should be considered in the differential diagnosis. Pyogenic infections are more aggressive, and intervertebral disc destruction is in the foreground. Paravertebral soft tissue mass is more common in TB spondylodiscitis [40].

\section{Tuberculous arthritis}

Tuberculous arthritis is mainly observed in weight-bearing joints such as the sacroiliac (Figs. 47, 48), hips, and knees and involves a single joint at a rate of $90 \%$. Radiographic findings include periarticular/juxta-articular osteoporosis, peripherally located marginal bone erosion, and progressive joint space narrowing, known as the triad of Phemister (Figs. 49, 50). Ankylosis and degeneration are also observed later $[3,23]$.

Although there is significant synovitis in early-stage TB arthritis, the articular cartilage remains intact [23]. Periarticular soft tissue calcification and abscesses can also be observed [23, 51].

\section{Tuberculous osteomyelitis}

Tuberculous osteomyelitis affects the femur, tibia, bones of hands and feet $[51,52]$. Plain film X-ray shows osteopenia in the metaphysis surrounded by lytic lesions with minimal sclerosis (Fig. 51) [53]. MRI imaging findings are variable appearance similar to pyogenic osteomyelitis (low T1, high T2) and revealed earlier than CT and X-ray [54]. Marrow edema (high T2), intraosseous abscesses (T2 hyperintense center (caseous center) and peripheral enhancement on T1), abnormalities of adjacent muscles and extraosseous soft tissues (such as edema, abscess, fistula) may be seen (Fig. 52, 53, 54) [54, 55]. The spread of infection to the epiphysis in children is a typical feature that helps distinguish TB from pyogenic infection [20]. In children, fusiform soft tissue swelling in the short tubular bones of the hands and feet that may accompany periostitis is consistent with tuberculous dactylitis [3, 40,51]. A balloon-like swelling and a cyst-like cavity appearance in the involved bone are known as "spina ventosa" (a wind-filled sail) $[40,56]$.

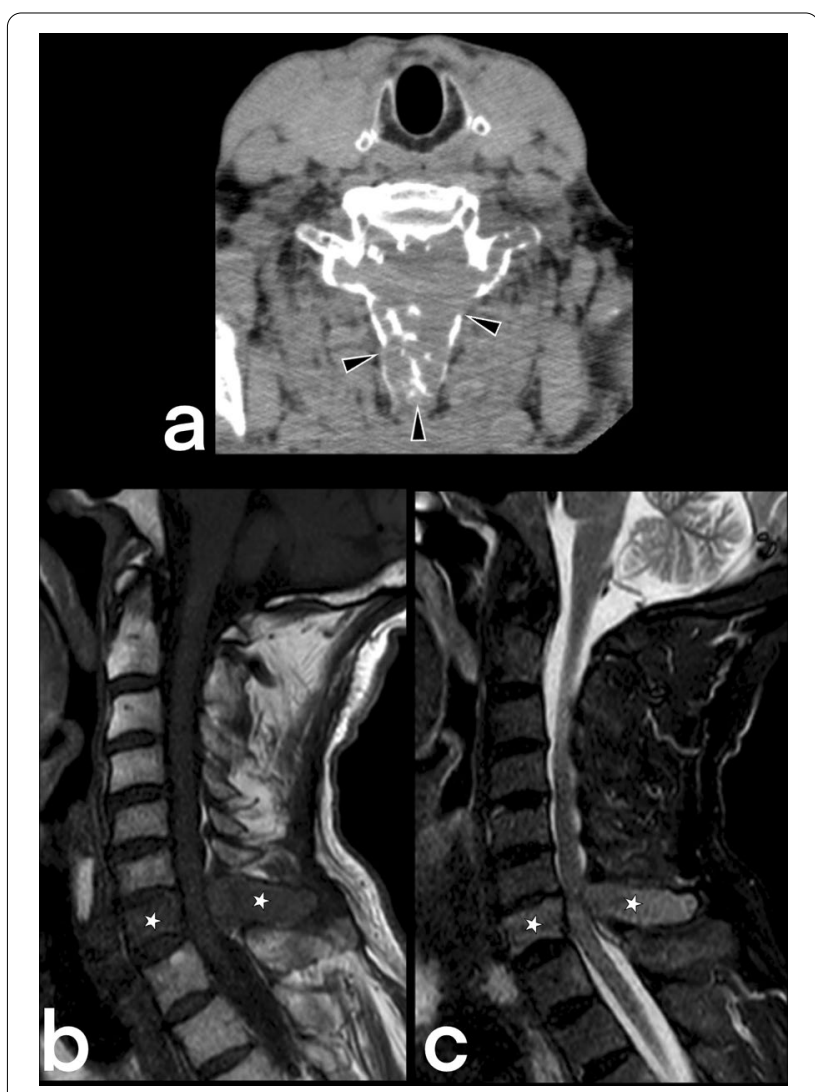

Fig. 46 A 41-year-old male with pain in the posterior neck region. Non-contrast axial CT image (a) demonstrates destruction and bone erosion of the posterior spinous process of the $\mathrm{C} 7$ vertebra (arrowheads). Pre-contrast sagittal T1 weighted image $(\mathbf{b})$ reveals diffuse decreased signal intensity within the $c 7$ vertebral body and posterior spinous process. The lesion shows prominent diffuse contrast enhancement on sagittal (c) T1-weighted images (stars)

\section{Muscle, skin and subcutaneous fat tissue TB}

Muscle, skin, and subcutaneous adipose tissue involvements are very rare [52]. In patients with tuberculous arthritis/osteomyelitis, the disease can spread through ruptured necrotic lymph nodes or from adjacent foci [51, 52]. Psoas abscesses (Fig. 55) usually develop secondary to spondylodiscitis [53]. Notable edema, myositis, or cellulitis is not observed around the lesion in muscle involvement (Fig. 56) [23]. Skin TB is seen as a result of exogenous inoculation, spreading from the adjacent foci of infection or through the hematogenous route. Cutaneous TB presents in different forms and can be seen as patches, plaque, macules, papules, nodules, abscesses, erosions, and ulcers [57]. 


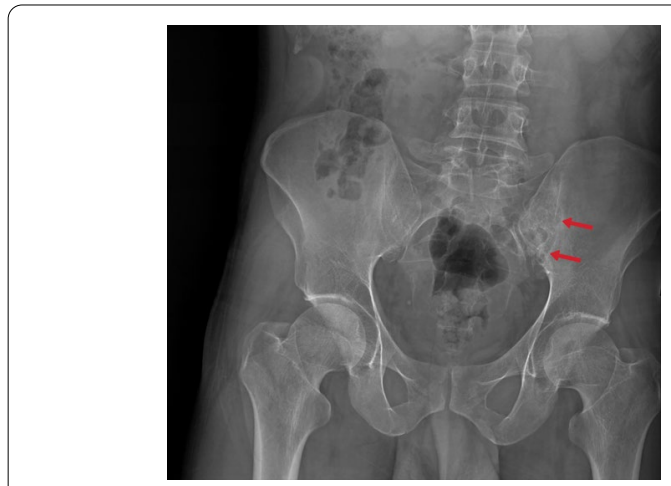

Fig. 47 Pelvic $x$-ray of a 46-year-old male. The late finding of tuberculous sacroiliitis is characterized by degenerative changes in the left sacroiliac joint (arrows)

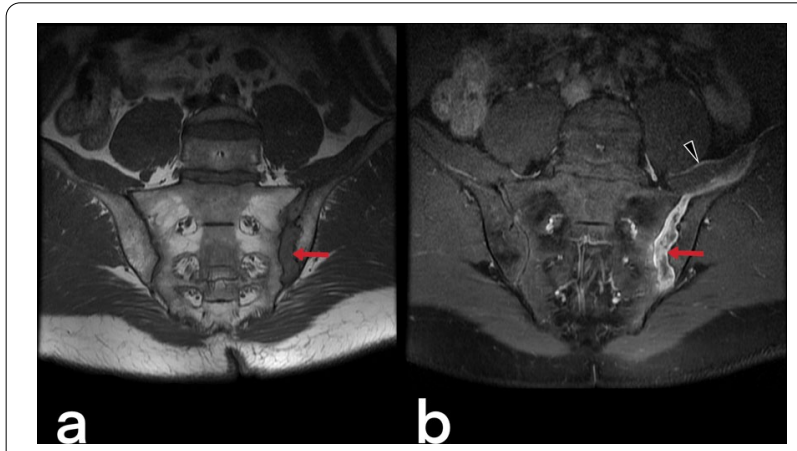

Fig. 48 MR images of a 43-year-old male. Pre-contrast T1 weighted (a) and post-contrast (b) T1 weighted images demonstrate contrast enhancement in the synovium of the left sacroiliac joint in keeping with acute sacroiliitis (arrows). Arrowhead shows an abscess formation in the left iliac muscle that enhances peripherally after contrast injection

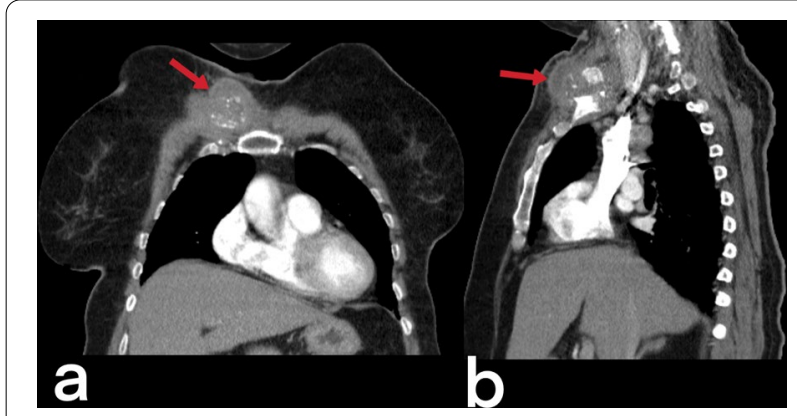

Fig. 49 Coronal (a) and sagittal (b) reformatted CT images of a 70-year-old female who has a complaint of swelling in the anterior wall of the chest. Both images reveal mass-like lesions in the right sternoclavicular joint, causing bone destruction containing small calcified areas. The patient was diagnosed with TB after the biopsy

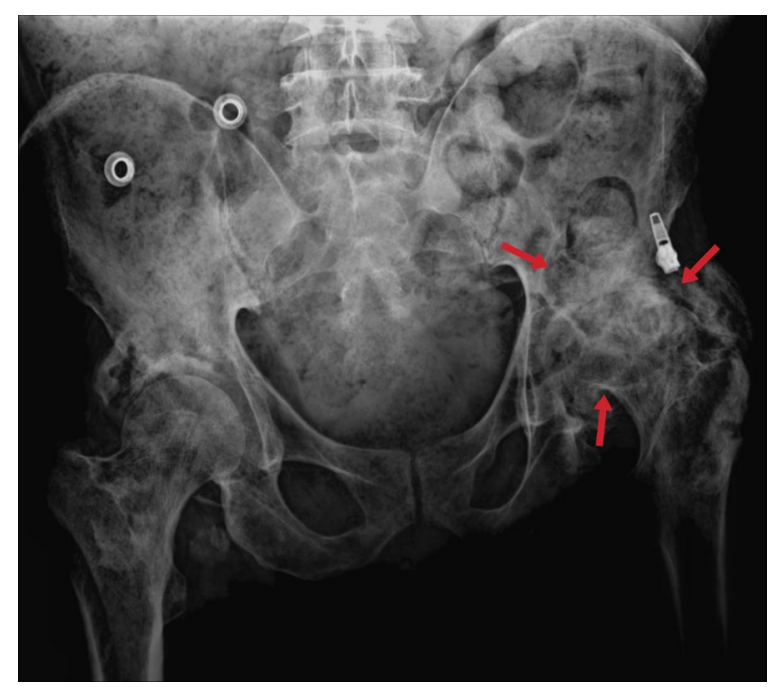

Fig. 50 Pelvic X-ray of a 53-year-old female. The left hip joint sequela of TB is characterized by degenerative arthritis (arrows)

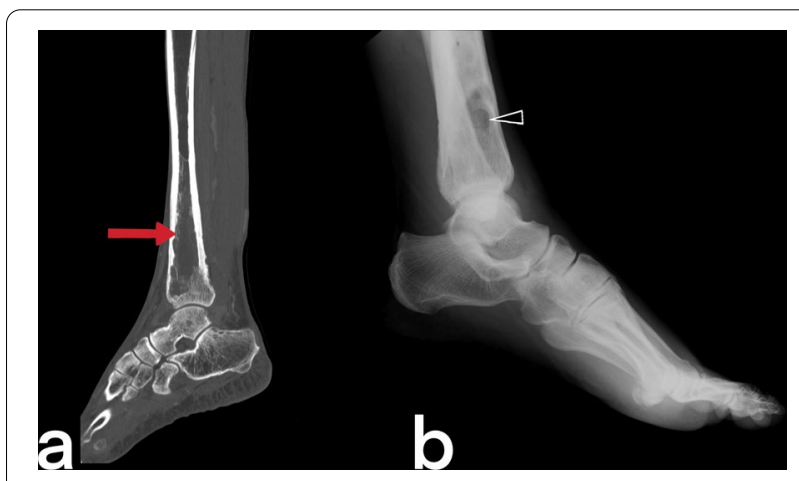

Fig. $51 \mathrm{CT}(\mathbf{a})$ and lateral ankle direct X-ray (b) images of two different patients. Lytic areas are observed in the tibia in both cases with proven tuberculous osteomyelitis and abscess

\section{Treatment and follow-up}

There are no medical treatment guidelines specific to EPTB. As in pulmonary TB treatment, EPTB is treated by 6-18 months of antitubercular chemotherapy. In tuberculous meningitis, corticosteroids are often used in addition to antitubercular chemotherapy, which can also help prevent fibrotic sequelae, including constrictive pericarditis and stenosis that may develop in hollow internal organs such as the intestine and ureter $[7$, 58]. 


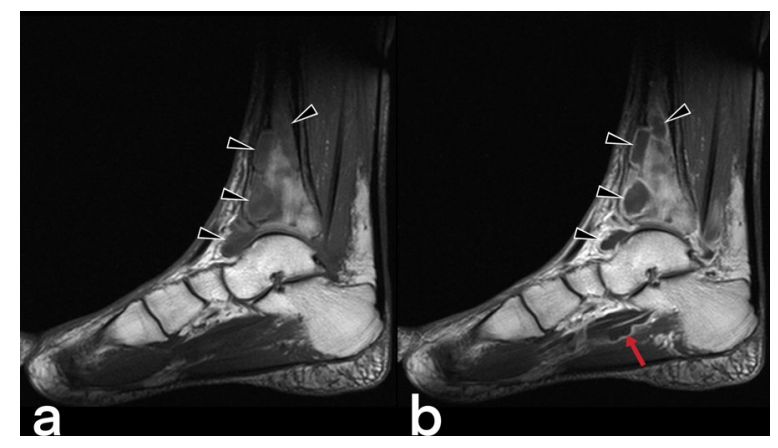

Fig. 52 MR images of a 59-year-old male. Pre-contrast (a) and postcontrast (b) T1 weighted images demonstrate intraosseous (arrowheads) and soft tissue abscesses (arrow) in the distal part of the tibia and plantar face of the foot

In a patient that recovers from the disease after treatment, TB lesions may reduce clinically or radiologically, or new lesions may develop a phenomenon known as a paradoxical reaction. Paradoxical reactions occur more frequently in EPTB than in pulmonary TB; lymph nodes, the pleura, and the CNS are the most frequently affected areas [58].

Surgery (such as lumpectomy or mastectomy in breast tuberculosis, surgical repair of kyphosis sequela in spinal

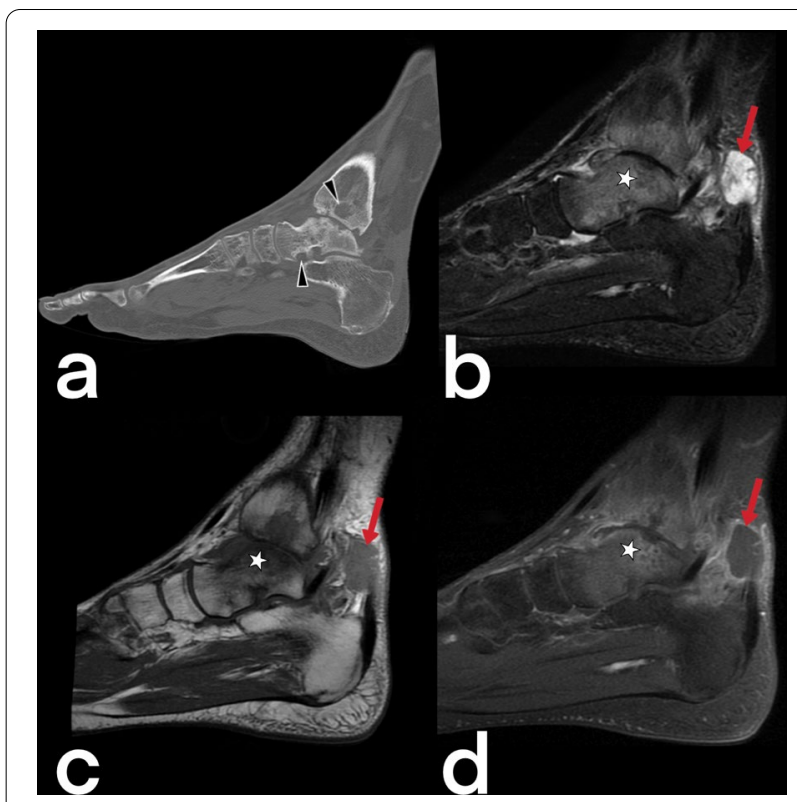

Fig. 53 Sagittal reformatted CT (a) and MR images (b-d) of a 48-year-old male. CT image shows focal low-attenuation areas in the distal part of the tibia in keeping with abscesses and bone destructions (arrowheads). On sagittal T2-weighted (b), pre-contrast T1-weighted (c), and post-contrast T1 weighted (d) MR images reveal extensive signal intensity change within the bone marrow of the talus and distal tibial part (stars). MR images also demonstrate an abscess in the adjacent soft tissue to the Achilles tendon (arrows)

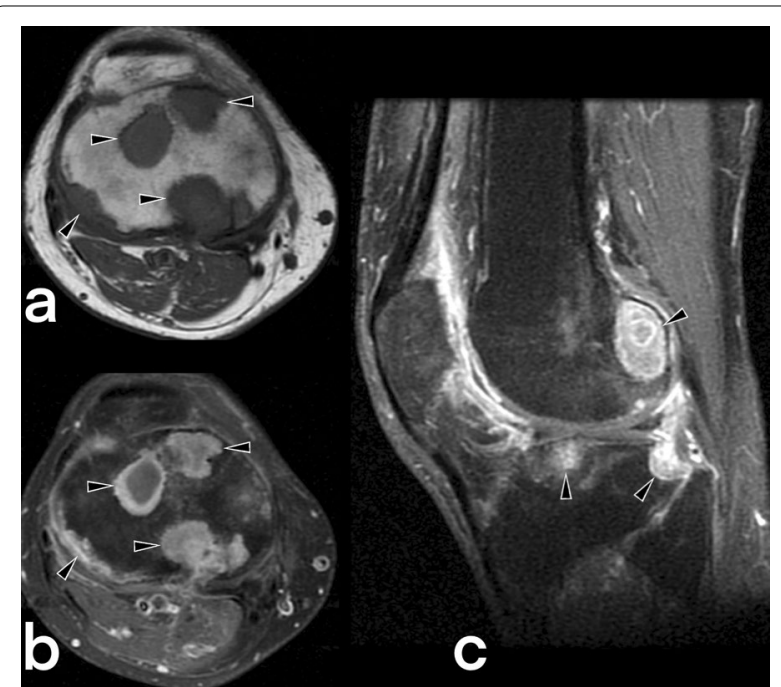

Fig. 54 MR images of a 60-year-old male. Pre-contrast axial (a), post-contrast axial (b), and sagittal (c) T1 weighted images reveal intraosseous abscesses (arrowheads) with peripheral enhancement in the distal part of the femur and proximal tibia

tuberculosis, a non-functioning kidney nephrectomy, or

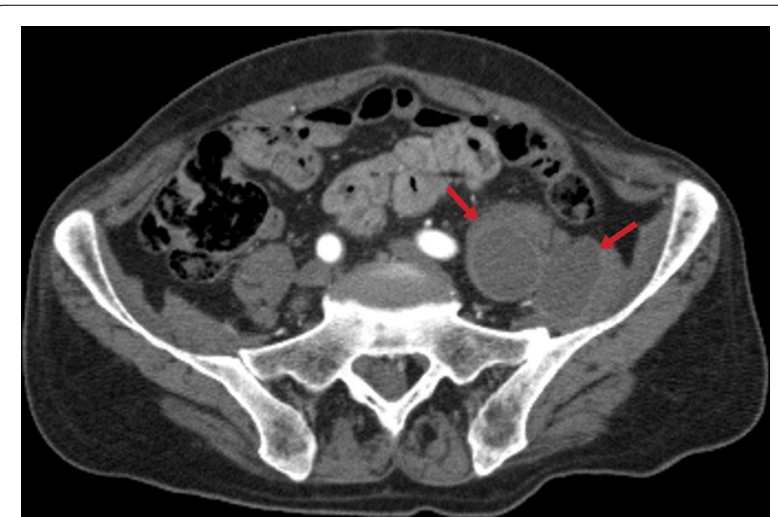

Fig. 55 A contrast-enhanced CT image of a 69-year-old male demonstrates the left iliopsoas abscess (arrows)

placement of a ventriculoperitoneal shunt for the treatment of hydrocephalus) is an option when there is no response to medical treatment. Abscesses can be treated effectively with US or CT-guided external drainage [7, 58].

For patients with EPTB, there is usually no need for a second biopsy or bacteriological evaluation to assess the response to treatment. The response should generally be evaluated based on clinical and radiological findings $[7$, 58]. 


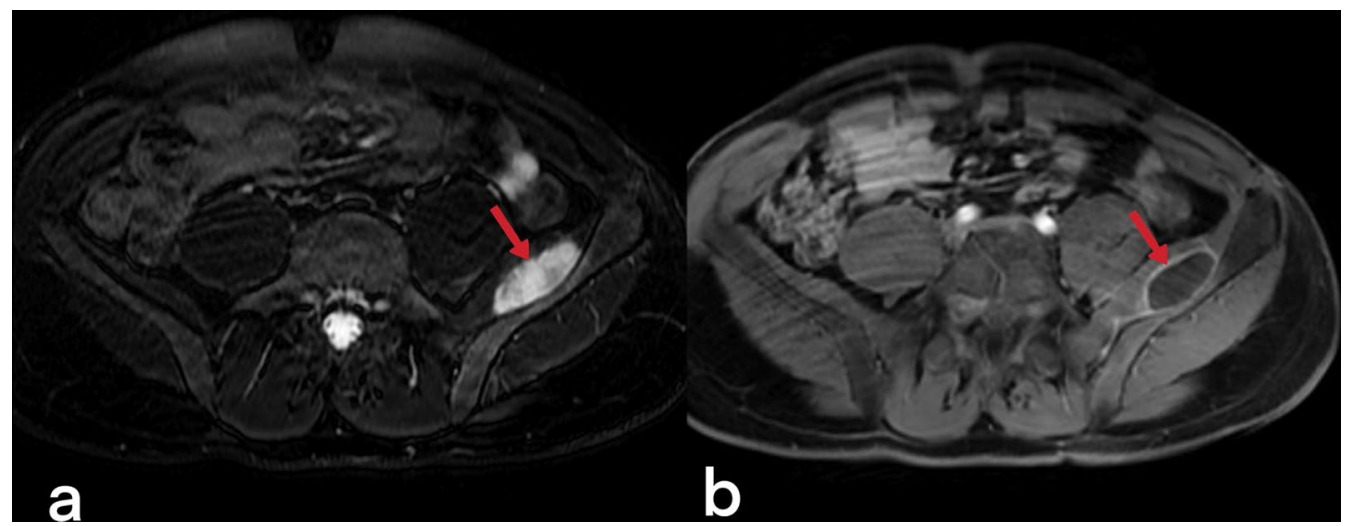

Fig. 56 T2 weighted (a) and post-contrast T1 weighted MR images of a 35-year-old male demonstrate abscess formation with peripheral enhancement in the left iliac muscle (b)

\section{Conclusion}

$\mathrm{TB}$ is a severe disease that can affect almost every organ and tissue of the body and should be considered in the differential diagnosis, particularly in patients with immune system disorders. Some radiological involvement patterns in EPTB are specific to the disease and can assist in early diagnosis. Knowing radiological findings can prevent unnecessary biopsy (such as brain biopsy) and surgical procedures that may result in organ loss (such as mastectomy, oophorectomy, splenectomy). Early diagnosis and treatment are the most critical factors in reducing morbidity associated with the disease.

\section{Abbreviations}

CNS: Central nervous system; CT: Computed tomography; EPTB: Extrapulmonary tuberculosis; MRI: Magnetic resonance imaging; TB: Tuberculosis.

\section{Acknowledgements}

The authors are grateful to M. Ece Baykan for contributing to the schematic diagram and figures design.

\section{Authors' contributions}

Data collection: AHB, HSS, MK, II; manuscript writing: AHB, EA; review and final editing: all authors. All authors contributed to the elaboration, critical revision, and review of the intellectual content. All authors read and approved the final manuscript.

\section{Funding}

The authors declare no funding was obtained for this paper.

\section{Availability of data and materials}

Not applicable.

\section{Declarations}

Ethics approval and consent to participate

Not applicable.

\section{Consent for publication}

Not applicable.

\section{Competing interests}

Mehmet Sukru Erturk is member of the Insights into Imaging Editorial Board. He has not taken part in the review or selection process of this article. The remaining authors declare that they have no competing interests.

\section{Author details}

${ }^{1}$ Department of Radiology, Faculty of Medicine, Adiyaman University, Yunus Emre Mahallesi 1164 Sokak No:13, 02200 Merkez, Adıyaman, Turkey. ${ }^{2}$ Department of Infectious Diseases and Clinical Microbiology, Faculty of Medicine, Adiyaman University, Adiyaman, Turkey. ${ }^{3}$ Department of Radiology, Izmir Tepecik Training and Research Hospital, Izmir, Turkey. ${ }^{4}$ Department of Radiology, Faculty of Medicine, Firat University, Elazig, Turkey. ${ }^{5}$ Department of Radiology" King's College Hospital London, Dubai, United Arab Emirates. ${ }^{6}$ Department of Radiology, Faculty of Medicine, Istanbul University, Istanbul, Turkey.

Received: 22 December 2021 Accepted: 3 February 2022 Published online: 07 March 2022

\section{References}

1. Heye T, Stoijkovic M, Kauczor H, Junghanss T, Hosch W (2011) Extrapulmonary tuberculosis: radiological imaging of an almost forgotten transformation artist. RoFo 183(11):1019-1029

2. World Health Organization. Global tuberculosis report. 2021. https:// www.who.int/teams/global-tuberculosis-programme/tb-reports/ global-tuberculosis-report-2021. Accessed 14 Oct 2021.

3. Gambhir S, Ravina M, Rangan K, Dixit M, Barai S, Bomanji J (2017) Imaging in extrapulmonary tuberculosis. Int J Infect Dis 56:237-247

4. Sharma SK, Mohan A, Kohli M (2021) Extrapulmonary tuberculosis. Expert Rev Respir Med 15(7):931-948

5. Sharma SK, Mohan A (2004) Extrapulmonary tuberculosis. Indian J Med Res 120(4):316-353

6. Harisinghani MG, MCLoud TC, Shepard J-AO, Kao JP, Shroff MM, Mueller PR (2000) Tuberculosis from Head to Toe 1: (CME available in print version and on RSNA link). Radiographics 20(2):449-470

7. Baykan AH, Sayiner HS, Inan I, Aydin E, Erturk SM (2021) Primary breast tuberculosis: imaging findings of a rare disease. Insights Imaging 12(1):1-11

8. Lawn S, Zumla A (2011) Tuberculosis. Lancet 378(9785):57-72

9. Hopwell PC, Barry R. Tuberculosis and other mycobacterial disease. Textbook of respiratory medicine 1994:1094-1160.

10. Brennan PJ (2003) Structure, function, and biogenesis of the cell wall of Mycobacterium tuberculosis. Tuberculosis 83(1-3):91-97

11. Muneer A, Macrae B, Krishnamoorthy S, Zumla A (2019) Urogenital tuberculosis-epidemiology, pathogenesis and clinical features. Nat Rev Urol 16(10):573-598 
12. Behr MA, Waters WR (2014) Is tuberculosis a lymphatic disease with a pulmonary portal? Lancet Infect Dis 14(3):250-255

13. Abengozar-Muela M, Esparza MV, Garcia-Ros D et al (2020) Diverse immune environments in human lung tuberculosis granulomas assessed by quantitative multiplexed immunofluorescence. Mod Pathol 33(12):2507-2519

14. Perez-Velez CM, Roya-Pabon CL, Marais BJ (2017) A systematic approach to diagnosing intra-thoracic tuberculosis in children. J Infect 74:S74-S83

15. Hopewell PC (1994) Overview of clinical tuberculosis. Tuberculosis:pathogenesis, protection, and control 1994:25-46

16. Das S, Das D, Bhuyan UT, Saikia N (2016) Head and neck tuberculosis: scenario in a tertiary care hospital of North Eastern India. J Clin Diagn Res 10(1):07. https://doi.org/10.7860/jcdr/2016/17171.7076

17. Bernaerts A, Vanhoenacker F, Parizel P et al (2003) Tuberculosis of the central nervous system: overview of neuroradiological findings. Eur Radiol 13(8):1876-1890

18. Thwaites GE, Hien TT (2005) Tuberculous meningitis: many questions, too few answers. Lancet Neurol 4(3):160-170

19. Natarajan A, Beena P, Devnikar AV, Mali S (2020) A systemic review on tuberculosis. Indian J Tuberc 67:295-311

20. Burrill J, Williams CJ, Bain G, Conder G, Hine AL, Misra RR (2007) Tuberculosis: a radiologic review. Radiographics 27(5):1255-1273

21. Morgado C, Ruivo N (2005) Imaging meningo-encephalic tuberculosis. Eur J Radiol 55(2):188-192

22. Poptani H, Gupta RK, Roy R, Pandey R, Jain VK, Chhabra DK (1995) Characterization of intracranial mass lesions with in vivo proton MR spectroscopy. AJNR Am J Neuroradiol 16(8):1593-1603

23. Rodriguez-Takeuchi SY, Renjifo ME, Medina FJ (2019) Extrapulmonary tuberculosis: pathophysiology and imaging findings. Radiographics 39(7):2023-2037

24. Marais S, Roos I, Mitha A, Mabusha SJ, Patel V, Bhigjee Al (2018) Spinal tuberculosis: clinicoradiological findings in 274 patients. Clin Infect Dis 67(1):89-98

25. Liu Y, Wang F, Xu J, Guan Y, Guan H (2010) Intramedullary thoracic tuberculoma. Spinal Cord 48(1):80-82

26. Sardana V, Shringi P (2019) Intramedullary tuberculoma of the spinal cord, clinical features \& imaging: possibility of early diagnosis with imaging? Indian J Tuberc 67(3):346-348

27. Thomas N, Nambiar SS, Nampoothiri PM (2021) Extrapulmonary tuberculosis: an otorhinolaryngologist's perspective. Indian J Otolaryngol Head Neck Surg 2021:1-7

28. De Backer A, Mortelé K, Van Den Heuvel E, Vanschoubroeck I, Kockx M, Van de Vyvere M (2007) Tuberculous adenitis: comparison of CT and MRI findings with histopathological features. Eur Radiol 17(4):1111-1117

29. Chou C-H, Yang T-L, Wang C-P (2014) Ultrasonographic features of tuberculous cervical lymphadenitis. J Med Ultrasound 22(3):158-163

30. Dhanasekar T, Shyamala RI, Arshad AM (2020) An interesting case of tonsillar tuberculosis in a 10-year-old boy. J Clin Tuberc Other Mycobact Dis 20:100162

31. Ortiz-Flores A, Gioia F, Montánez-Fernández L et al (2017) Incidental finding of a primary thyroid tuberculosis. Oxford Med Case Rep 2017(6):omx022

32. Cowley A, Dobson L, Kurian J, Saunderson C (2017) Acute myocarditis secondary to cardiac tuberculosis: a case report. Echo Res Pract 4(3):K25

33. Sakr AA, Fawzy RK, Fadaly G, Baky MA (2004) Mammographic and sonographic features of tuberculous mastitis. Eur J Radiol 51(1):54-60

34. Tewari M, Shukla H (2005) Breast tuberculosis: diagnosis, clinical features \& management. Indian J Med Res 122(2):103

35. Thimmappa D, Mallikarjuna M, Vijayakumar A (2015) Breast tuberculosis. Indian J Surg 77(3):1378-1384

36. Ladumor H, Al-Mohannadi S, Ameerudeen FS, Ladumor S, Fadl S (2021) TB or not TB: a comprehensive review of imaging manifestations of abdominal tuberculosis and its mimics. Clin Imaging 76:130-143

37. Sharma M, Bhatia V (2004) Abdominal tuberculosis. Indian J Med Res 120:305-315

38. Debi U, RavisankarV, Prasad KK, Sinha SK, Sharma AK (2014) Abdominal tuberculosis of the gastrointestinal tract: revisited. World J Gastroenterol 20(40):14831
39. Karaosmanoglu AD, Onur MR, Sahani DV, Tabari A, Karcaaltincaba M (2016) Hepatobiliary tuberculosis: imaging findings. AJR Am J Roentgenol 207(4):694-704

40. Engin G, Acunas B, Acunas G, Tunaci M (2000) Imaging of extrapulmonary tuberculosis 1: (CME available in print version and on RSNA Link). Radiographics 20(2):471-488

41. Kakkar C, Polnaya AM, Koteshwara P, Smiti S, Rajagopal K, Arora A (2015) Hepatic tuberculosis: a multimodality imaging review. Insights Imaging 6(6):647-658

42. Vanhoenacker F, De Backer A, de Beeck BO et al (2004) Imaging of gastrointestinal and abdominal tuberculosis. Eur Radiol Suppl 14(3):E103-E115

43. Melo VA, Melo GB, Silva RL, Piva N, Almeida MLD (2004) Tuberculosis of the cystic duct lymph node. Braz J Infectious Dis 8:112-114

44. Golden MP, Vikram HR (2005) Extrapulmonary tuberculosis: an overview. Am Fam Physician 72(9):1761-1768

45. Gibson MS, Puckett ML, Shelly ME (2004) Renal tuberculosis. Radiographics 24(1):251-256

46. Grace GA, Devaleenal DB, Natrajan M (2017) Genital tuberculosis in females. Indian J Med Res 145(4):425

47. Jung YY, Kim JK, Cho K-S (2005) Genitourinary tuberculosis: comprehensive cross-sectional imaging. AJR Am J Roentgenol 184(1):143-150

48. Naeem M, Zulfiqar M, Siddiqui MA et al (2021) Imaging manifestations of genitourinary tuberculosis. Radiographics 41:1123-1143

49. Merchant S, Bharati A, Merchant N (2013) Tuberculosis of the genitourinary system-urinary tract tuberculosis: renal tuberculosis-part I. Indian J Radiol Imaging 23(1):46

50. Lenk S, Schroeder J (2001) Genitourinary tuberculosis. Curr Opin Urol 11(1):93-96

51. De Vuyst D, Vanhoenacker F, Gielen J, Bernaerts A, De Schepper AM (2003) Imaging features of musculoskeletal tuberculosis. Eur Radiol 13(8):1809-1819

52. Leonard MK Jr, Blumberg HM (2017) Musculoskeletal tuberculosis. Microbiol Spectrum 5(2):5-2

53. Griffith JF, Kumta SM, Leung PC, Cheng JC, Chow LT, Metreweli C (2002) Imaging of musculoskeletal tuberculosis: a new look at an old disease. Clin Orthop Relat Res 398:32-39

54. Shikhare SN, Singh DR, Shimpi TR, Peh WC. Tuberculous osteomyelitis and spondylodiscitis. Seminars in musculoskeletal radiology: @ Thieme Medical Publishers, 2011; p. 446-58.

55. Sharma P (2003) MR features of tuberculous osteomyelitis. Skeletal Radiol 32(5):279-285

56. Vanhoenacker FM, Sanghvi DA, De Backer Al (2009) Imaging features of extraaxial musculoskeletal tuberculosis. Indian J Radiol Imaging 19(3):176

57. Chen Q, Chen W, Hao F (2019) Cutaneous tuberculosis: a great imitator. Clin Dermatol 37(3):192-199

58. Lee JY (2015) Diagnosis and treatment of extrapulmonary tuberculosis. Tuberc Respir Dis 78(2):47-55

\section{Publisher's Note}

Springer Nature remains neutral with regard to jurisdictional claims in published maps and institutional affiliations.

\section{Submit your manuscript to a SpringerOpen ${ }^{\circ}$ journal and benefit from:}

- Convenient online submission

- Rigorous peer review

- Open access: articles freely available online

- High visibility within the field

- Retaining the copyright to your article

Submit your next manuscript at $\boldsymbol{\nabla}$ springeropen.com 